\title{
Electron-photon coupling in Mesoscopic Quantum Electrodynamics
}

\author{
A. Cottet $^{1}$, T. Kontos ${ }^{1}$ and B. Doucot ${ }^{2}$ \\ ${ }^{1}$ Laboratoire Pierre Aigrain, Ecole Normale Supérieure-PSL Research University, \\ CNRS, Université Pierre et Marie Curie-Sorbonne Universités, \\ Université Paris Diderot-Sorbonne Paris Cité, \\ 24 rue Lhomond, 75231 Paris Cedex 05, France and \\ ${ }^{2}$ Sorbonne Universités, Université Pierre et Marie Curie, CNRS, \\ LPTHE, UMR 7589, \& place Jussieu, 75252 Paris Cedex 05
}

(Dated: May 12, 2015)

\begin{abstract}
Understanding the interaction between cavity photons and electronic nanocircuits is crucial for the development of Mesoscopic Quantum Electrodynamics (QED). One has to combine ingredients from atomic Cavity QED, like orbital degrees of freedom, with tunneling physics and strong cavity field inhomogeneities, specific to superconducting circuit QED. It is therefore necessary to introduce a formalism which bridges between these two domains. We develop a general method based on a photonic pseudo-potential to describe the electric coupling between electrons in a nanocircuit and cavity photons. In this picture, photons can induce simultaneously orbital energy shifts, tunneling, and local orbital transitions. We study in details the elementary example of a single quantum dot with a single normal metal reservoir, coupled to a cavity. Photon-induced tunneling terms lead to a non-universal relation between the cavity frequency pull and the damping pull. Our formalism can also be applied to multi quantum dot circuits, molecular circuits, quantum point contacts, metallic tunnel junctions, and superconducting nanostructures enclosing Andreev bound states or Majorana bound states, for instance.
\end{abstract}

\section{INTRODUCTION}

Cavity Quantum ElectroDynamics (Cavity QED) enables the study of the interaction between light and matter at the most elementary level, thanks to the achievement of a strong coupling between a single atom and a single photon trapped in a microwave or optical cavity $\underline{\underline{1}}$. This paradigm has been recently brought into superconducting circuits: artificial atoms consisting of two level superconducting circuits have been coupled to superconducting cavities ${ }^{2,3}$, in the context of Circuit QED. These experiments provide an ideal playground to test the basic laws of quantum mechanics because they can be described in terms of simple models like the JaynesCummings Hamiltonian. However, such models conceal essential physical differences between Cavity and Circuit QED. On the one hand, the coupling between isolated atoms and cavity photons mainly occurs due to the sensitivity of the atom electric dipole to the cavity electric field. This coupling depends on microscopic details since the atomic dipole is set by the structure of the atom electronic orbitals. Furthermore, one can generally perform the "electric-dipole approximation" which assumes that the cavity field varies little on the scale of the atomic system ${ }^{4}$. On the other hand, the behavior of submicronic superconducting circuits is essentially insensitive to microscopic details due to the rigidity of the superconducting phase $\mathrm{e}^{5}$. For instance, the behavior of a superconducting charge qubit can be described with one macroscopic variable, i.e. the total charge of a superconducting island $\mathrm{d}^{\mathbf{7}}$. This charge can vary due to the Josephson coupling between the island and an external superconducting reservoir. The coupling between the superconducting charge qubit and the cavity is usually de- scribed in terms of a capacitive coupling between the superconducting island and the cavity central conductor. As a result, the chemical potential of the superconducting island is shifted proportionally to the cavity electric potentia ${ }^{8}$. This picture implies strong inhomogeneities of the photonic electric field on the scale of the superconducting qubit, in contrast to what is generally considered in atomic Cavity QED for a single atom.

Recent technological progress is enabling the development of a new type of experiments where nanocircuits based on carbon nanotubes, semiconducting nanowires, two-dimensional electron gases or graphene, are coupled to coplanar microwave cavities $\underline{\underline{-20}}$. This paves the way for the development of "Mesoscopic QED", a denomination introduced in a pioneering theory work ${ }^{21}$. Mesoscopic QED opens many possibilities because nanoconductors can be tunnel-coupled to various types of fermionic reservoirs such as normal metals, ferromagnets 22 or superconductors ${ }^{23}$, in a large variety of geometries. So far, theoretical studies on Mesoscopic QED have mainly focused on quantum dot circuits $21,24 \underline{43}$. Several configurations have been suggested to reach the strong coupling regime between an electronic spin and cavity photons ${ }^{24-29}$, or more generally, to develop quantum computing schemes ${ }^{44}-52$. Mesoscopic QED also tackles problems which go beyond the mechanics of closed two level systems coupled to cavities, usually considered in Cavity or Circuit QED. The interaction between electronic transport and the light-matter interaction leads to a rich phenomenology 21,31-42,53,54. Besides, coplanar cavities could be used as a powerful probe to reveal some exotic properties of hybrid nanocircuits, like for instance the existence of topological superconducting phases $\frac{55}{5}$, Majorana quasiparticle modes $44-52,56$, or spin-entanglement in a Cooper pair 
beam splitter 41,42 . On the experimental side, pioneering works have focused on mesoscopic rings 57 and metallic tunnel junctions ${ }^{58}$. More recently, experiments have been performed with single quantum dots 9,10 and double quantum dots (DQDs) ${ }^{11-20}$ with normal metal reservoirs. Reaching the strong coupling regime between the charge states of a DQD and a cavity is still a challenge ${ }^{59}$. Nevertheless, interesting resonance phenomena have already been observed $11-15,17$. Several experiments have also provided evidence for a modification of the cavity behavior by finite bias transport through a $\mathrm{DQD}^{17,19}$, including a maser effect ${ }^{20}$.

These recent developments call for a full description of the coupling between a hybrid nanocircuit and cavity photons. One question naturally arises: is Mesoscopic QED closer to atomic Cavity QED or superconducting Circuit QED? What are the specificities of the coupling between a nanocircuit and a cavity? So far, most theory works have considered a capacitive coupling between the nanocircuit and the cavity central conductor, by analogy with Circuit QED $27,30,31,33-40,43,54,56$. This approach implies a coarse grained electric description of the nanocircuit, and a concentration of the non-homogeneous photonic electric field inside some capacitive elements. A few works have considered a direct coupling between the motion of electrons trapped in the nanoconductors and the bare cavity electric field, which is assumed to be constant on the scale of the nanocircuit ${ }^{24-26,29,41,52}$. This is reminiscent of the descriptions used in Cavity QED ${ }^{4}$. In this article, we introduce a description of Mesoscopic QED which bridges between these two approaches. We use a model which focuses on conduction electrons tunneling between the different elements of a nanocircuit. The tunneling electrons occupy quasi-localized orbitals in each nanocircuit element, which recalls the atomic orbital degree of freedom of Cavity QED. However, there also exists collective plasmonic modes in the nanocircuit, which can screen at least partially the cavity fields. We use a gaugeinvariant Mesoscopic QED Hamiltonian which accounts for the non-uniform screening of the cavity fields inside the nanocircuit, and for the electromagnetic boundary conditions provided by cavity conductors and voltagebiased nanocircuit DC gates. In the limit where photoninduced magnetic effects are negligible, we can reexpress the Mesoscopic QED Hamiltonian in terms of a scalar photonic pseudo-potential. This picture unifies the different approaches used so far to describe Mesoscopic QED devices, since the photonic pseudo-potential can vary linearly with space in the case of a locally uniform photonic electric field (dipolar coupling limit), as well as remain constant inside a given circuit element in the limit of a coarse grained circuit model. In the framework of a tunneling model, the photonic pseudo-potential leads to various types of linear electron/photon coupling terms: cavity photons shift the orbital energies of the different nanocircuit elements, but also induce simultaneously tunneling and local orbital transitions. This general description can be used to study the behavior of many dif- ferent types of Mesoscopic QED devices. For instance, it can be applied to quantum dot circuits, molecular circuits, quantum point contacts, metallic tunnel junctions, and superconducting nanostructures enclosing Andreev bound states or Majorana bound states. To illustrate the richness of our approach, we study in details the elementary example of a cavity coupled to a "quantum RC circuit", i.e. a single quantum dot coupled to a single normal metal reservoir. The photon-induced tunneling terms between the quantum dot and the reservoir induce a non-universal relation between the cavity frequency pull and the cavity damping pull, contrarily to what is expected with purely capacitive coupling schemes at low temperatures $60-66$.

This paper is organized as follows. In section II A, we discuss the gauge-invariant Mesoscopic QED Hamiltonian, which involves a photonic vector potential. In section II B, we perform a unitary transformation to obtain a new Hamiltonian where the electron/photon coupling is due to the scalar photonic pseudo-potential. In section II C, we reexpress the photonic pseudo-potential scheme in the framework of a tunnelling model. In section II D, we discuss the application of our formalism to the case of nanocircuits with superconducting elements. In particular, we give an explicit Hamiltonian for a nanostructure with Majorana bound states coupled to a cavity. In section III, we work out in details the case of the quantum RC circuit coupled to a cavity. Section IV concludes. Appendix A gives a detailed mathematical justification for the form of the Hamiltonian of section II A, on the basis of an effective model which separates physically the tunneling electrons occupying individual orbital states in the different elements of a nanocircuit, from the plasmonic collective modes of this nanocircuit. Appendix B discusses the advantages of the photonic pseudo-potential scheme.

\section{GENERAL DESCRIPTION OF MESOSCOPIC QED}

\section{A. Gauge invariance and minimal coupling scheme}

A nanocircuit encloses a large variety of degrees of freedom. Our main interest in this paper is the interaction of the cavity with the ensemble $\mathcal{T}$ of the "tunneling" conduction electrons, which can occupy orbital states in the different elements of a nanocircuit and tunnel between them. However, there also exists plasmonic electronic modes which are responsive for the screening of external fields from a massive metallic electrode. Plasmons can also convey screening currents in the reservoir electrical lines, which reequilibrate locally charge distributions on tunnel junctions after a tunneling event. The question of plasmonic modes in a nanocircuit is complicated since nanoconductors have generally a low electronic density, which allows only a partial screening of the cavity field 67 . In principle, even the metallic con- 


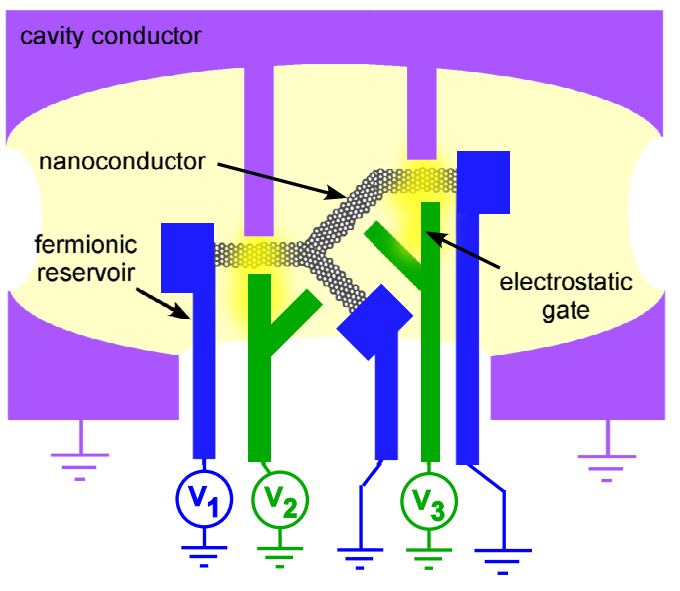

FIG. 1: Scheme of a loopless nanocircuit with source/drain electrodes (blue) and electrostatic gates (green) embedded in a photonic cavity (purple). The yellow cloud represents the photonic field. The cavity presents some protrusions (purple stripes) fabricated to increase the photonic field inhomogeneities (darker yellow areas).

tacts of the nanocircuit are not able to screen totally the cavity field due to their thinness. In the limit where the nanocircuit contacts are connected to outside electrical lines with a low impedance, it is reasonable to assume that the dynamics of plasmonic modes in the nanocircuit is very fast compared to the dynamics of the cavity modes and of the tunneling electrons $\frac{68}{}$. In this case, one can assume that the plasmonic modes in the nanocircuit generate a distribution of screening charges which is enslaved to the position of the tunneling charges and to the value of the cavity field. This produces a renormalization of the cavity field properties, and in particular its spatial dependence nearby and inside the nanocircuit. It also produces a renormalization of the Coulomb interactions between the tunneling charges. In general, the Coulomb interactions between the nanocircuit charges is also renormalized by the screening charges on the cavity conductors (see Appendix A). The positive ions and valence electrons constituting the crystalline structure of the nanocircuit can be treated in a mean field approach, leading to an effective potential $V_{\text {conf }}(\vec{r})$ which confines tunneling electrons inside the nanocircuit. This confinement potential can delimit quantum dots, or induce disorder effects in nanoconductors, for instance. This approach is sufficient provided the background charges producing $V_{\text {conf }}(\vec{r})$ and the microwave cavity are far off resonant. In this framework, gauge invariance imposes the Hamiltonian

$$
\hat{H}_{\text {tot }}=\int d^{3} r \hat{\psi}^{\dagger}(\vec{r}) \hat{h}_{\mathcal{T}}(\vec{r}) \hat{\psi}(\vec{r})+\hat{H}_{\text {Coul }}+\hbar \omega_{0} \hat{a}^{\dagger} \hat{a}
$$

with

$$
\begin{gathered}
\hat{h}_{\mathcal{T}}(\vec{r})=\frac{1}{2 m}\left(\frac{\hbar \vec{\nabla}_{\vec{r}}}{i}+e \overrightarrow{\hat{A}}(\vec{r})\right)^{2}-e \Phi_{\text {harm }}(\vec{r})-e V_{\text {conf }}(\vec{r}), \\
\hat{H}_{\text {Coul }}=\frac{e^{2}}{2} \int d^{3} r d^{3} r^{\prime} \hat{\psi}^{\dagger}(\vec{r}) \hat{\psi}^{\dagger}\left(\vec{r}^{\prime}\right) G\left(\vec{r}, \vec{r}^{\prime}\right) \hat{\psi}\left(\vec{r}^{\prime}\right) \hat{\psi}(\vec{r}) \\
\overrightarrow{\hat{A}}(\vec{r})=\overrightarrow{\mathcal{A}}(\vec{r}) i\left(\hat{a}-\hat{a}^{\dagger}\right)
\end{gathered}
$$

and $e>0$ the elementary charge. The field operator $\hat{\psi}(\vec{r})$ includes all the tunneling charges of the nanocircuit. Above, $\hat{h}_{\mathcal{T}}(\vec{r})$ is a single electron Hamiltonian, $G\left(\vec{r}, \vec{r}^{\prime}\right)$ describes the Coulomb interaction between the tunneling electrons (see Appendix A for details), and $\hbar \omega_{0} \hat{a}^{\dagger} \hat{a}$ is the Hamiltonian of a (renormalized) cavity electromagnetic mode. The potential $\Phi_{\text {harm }}(\vec{r})$ is due to DC voltages applied on the nanocircuit electrostatic gates and to constant charges on floating conductors like the cavity central conductor in a coplanar geometry. The coupling between the cavity and the tunneling electrons arises from the photonic vector potential term of Eq.(44). For simplicity, we have considered a single cavity mode with creation operator $\hat{a}^{\dagger}$ and vector potential profile $\overrightarrow{\mathcal{A}}(\vec{r})$. This description can be generalized straightforwardly to the multimode case by introducing a sum on a cavity mode index in Eqs. (1) and (4). Note that $\overrightarrow{\mathcal{A}}(\vec{r})$ can strongly vary on the scale of the nanocircuit.

The formal description of the electromagnetic field and the light/matter interaction in Mesoscopic QED requires some care. In atomic cavity QED, the cavity field is generally assumed to vary slowly on the scale of an atom ${ }^{4}$. In contrast, we have to take into account strong spatial variations of the photonic field on the scale of a nanocircuit. In particular, a protrusion of a cavity conductor can be used to increase the photonic field locally, close to a given quantum dot11,13,14,17 (See Fig. 1). The DC voltagebiased electrostatic gates, used to control the nanocircuit spectrum, provide supplementary boundary conditions on the electromagnetic field. Besides, we have already mentioned that the plasmonic screening charges on the nanocircuit conductors can modify the photonic vector potential profile, especially inside and around fermionic reservoirs. In order to describe this complex reality and justify mathematically the shape of Eqs.(114), we introduce in Appendix A an effective model which separates physically the tunneling electrons occupying individual orbital states in the nanocircuit from the collective plasmonic modes of the nanocircuit. In this framework, one can introduce a Hodge decomposition of the electromagnetic field ${ }^{69,70}$, which can be obtained thanks to the use of an auxiliary electrostatic Green's function. This leads to the terms in $\vec{A}(\vec{r}), G\left(\vec{r}, \vec{r}^{\prime}\right)$ and $\Phi_{\text {harm }}(\vec{r})$ in Eqs. (1.41).

In this section, we disregard the spin of charge carriers because we want to focus on regimes where charge 
effects prevail. The introduction of the spin degree of freedom in Hamiltonian (11) would be straightforward by invoking gauge invariance (see for instance section IE 1). The direct coupling between the particles spin and the photonic magnetic field can usually be disregarded because this coupling is expected to be very weak unless collective excitations are considered in a large ensemble of spins $21-\underline{73}$. From recent predictions, real $24-26$ or effective $\underline{27-29}$ spin-orbit coupling provide an alternative way to obtain a spin-photon coupling.

In Eq.(2), the coupling of tunneling electrons to cavity photons occurs through two terms, one in $\vec{\nabla} \vec{r} \cdot \vec{A}+\vec{A} \cdot \vec{\nabla} \vec{r}$, corresponding to single photon transitions, and the other one in $\hat{A}^{2}$, corresponding to two photon transitions. The second has no reason to be disregarded in the general case, even if it has no structure in the electronic sector. For instance, this term is crucial for determining the existence of a Dicke quantum phase transition when many two-level systems are coupled transversely to a cavity ${ }^{74.75}$. The $\widehat{A}^{2}$ term can also bring a non-negligible contribution to the cavity frequency pull caused by one nanocircuit, which is a central quantity in Mesoscopic QED experiments (see Appendix B). Hence, it is convenient to introduce a new representation in which the $\hat{A}^{2}$ term is eliminated. This task is completed in the next section.

\section{B. Photonic pseudo-potential scheme}

In atomic Cavity QED, the Power-Zienau-Wooley (PZW) transformation formally enables the elimination of a space-dependent $\hat{A}(\vec{r})^{2}$ term from the system Hamiltonian ${ }^{4}$. This generalizes the widely known "electric-dipole" transformation used in the case where $\hat{A}(\vec{r})$ can be considered as constant on the scale of an atom. However, the natural variables of the PZW Hamiltonian are polarization and magnetization densities associated to the charge distribution of an atomic system, which are not directly useful in our case. To reexpress the PZW Hamiltonian in terms of particles coordinates and momenta, it is necessary to perform a multipolar development of the light/matter interaction ${ }^{4}$. In practice, this development is performed to a limited order, which means that only a moderate space dependence of the photonic electric and magnetic fields is taken into account. In this section, we perform a unitary transformation of Hamiltonian (11) in the same spirit as the PZW transformation, but with specificities required for Mesoscopic QED. Upon disregarding magnetic effects, we can take into account a strong space dependence of the photonic electric field, thanks to the use of a scalar photonic pseudo-potential. This procedure avoids the development of the light/matter interaction in terms of multipoles. This is necessary to describe e.g. multiquantum dot circuits with strongly asymmetric capacitive couplings to the cavity. Furthermore, we use a second-quantized description of charges, which is more convenient to describe central phenomena of mesoscopic physics like tunneling but also superconductivity (see next section).

To account for the possibly strong spatial variations of $\overrightarrow{\mathcal{A}}(\vec{r})$ on the scale of a nanocircuit, we define inside the volume of the nanocircuit a reference point $\vec{r}_{0}$ and a continuous functional path $C\left(\vec{r}, \vec{r}^{\prime}\right)$ relating any points $\vec{r}$ and $\vec{r}^{\prime}$. We also define a photonic pseudo-potential $V_{\perp}(\vec{r})\left(\hat{a}+\hat{a}^{\dagger}\right)$ with

$$
V_{\perp}(\vec{r})=\omega_{0} \int_{C\left(\vec{r}_{0}, \vec{r}\right)} \overrightarrow{\mathcal{A}}\left(\vec{r}^{\prime}\right) \cdot \overrightarrow{d \ell} .
$$

Importantly, one can use $\vec{\nabla}_{\vec{r}} \cdot V_{\perp}(\vec{r}) \simeq \omega_{0} \overrightarrow{\mathcal{A}}(\vec{r})$ inside the nanocircuit volume, provided the flux of $\vec{\nabla}_{\vec{r}} \wedge \overrightarrow{\mathcal{A}}$ through the nanocircuit can be disregarded. This criterion is generally satisfied, considering the typical amplitude of the cavity magnetic field and the size of nanoconductors. In this case, the arbitrariness on the choice of the contours $C\left(\vec{r}, \vec{r}^{\prime}\right)$ leads only to marginal effects. One exception is nanocircuits with large loops leading to magnetic Aharonov-Bohm effects. This case is beyond the scope of the rest of this article, which focuses on photon-induced electric effects. We perform the transformation

$$
\widetilde{H_{t o t}}=\mathcal{U}^{\dagger} H_{t o t} \mathcal{U}
$$

with

$$
\mathcal{U}=\exp \left(\frac{e\left(\hat{a}-\hat{a}^{\dagger}\right)}{\hbar \omega_{0}} \int d^{3} r V_{\perp}(\vec{r}) \hat{\psi}^{\dagger}(\vec{r}) \hat{\psi}(\vec{r})\right) .
$$

This gives

$$
\begin{aligned}
\widetilde{H_{\text {tot }}} & =\int d^{3} r \hat{\psi}^{\dagger}(r) \widetilde{h}_{\mathcal{T}}(\vec{r}) \hat{\psi}(\vec{r})+\hat{H}_{\text {Coul }}+\hbar \omega_{0} \hat{a}^{\dagger} \hat{a} \\
& +\hat{\mathcal{V}}\left(\hat{a}+\hat{a}^{\dagger}\right)+\left(\hat{\mathcal{V}}^{2} / \hbar \omega_{0}\right)
\end{aligned}
$$

with

$$
\hat{\mathcal{V}}=-e \int d^{3} r V_{\perp}(\vec{r}) \hat{\psi}^{\dagger}(\vec{r}) \hat{\psi}(\vec{r})
$$

and $\widetilde{h}_{\mathcal{T}}(\vec{r})=-\hbar^{2} \Delta_{\vec{r}} / 2 m-e \Phi_{\text {harm }}(\vec{r})-e V_{\text {conf }}(\vec{r})$. In this new representation, the single electron potential and the Coulomb interactions are renormalized by the term in $\hat{\mathcal{V}}^{2}$, but the electron/photon coupling takes a simpler linear form. The motion of tunneling electrons is modified by the pseudo-potential $V_{\perp}(\vec{r})\left(\hat{a}+\hat{a}^{\dagger}\right)$. Note that the arbitrariness on the choice of $\vec{r}_{0}$ is not an issue, since a change in $\vec{r}_{0}$ shifts $V_{\perp}(\vec{r})$ by a global constant and leads to a unitary transformation of $\widetilde{\hat{H}}_{t o t}$. In the limit where the spatial dependence of the photonic vector potential can be disregarded i.e. $\vec{A}(\vec{r}) \simeq \vec{A}_{0}$, one finds $V_{\perp}(\vec{r})=\vec{E}_{0} \cdot \vec{r}\left(\hat{a}+\hat{a}^{\dagger}\right)$ with $\vec{E}_{0}(\vec{r})=\omega_{0} \vec{A}_{0}$. This corresponds to the electric-dipole approximation frequently used in atomic Cavity QED. In the opposite limit of a 
small enough conductor or a perfect screening of $\vec{A}(\vec{r})$ inside this conductor, one can use a $V_{\perp}(\vec{r})$ constant inside this conductor. This is reminiscent of the capacitive network models frequently used to describe superconducting charge qubits in Circuit QED. Note that we do not assume that the ensemble $\mathcal{T}$ of the tunneling charges is neutral.

\section{Tunneling model and coarse graining of nanocircuits}

In this section, we consider a nanocircuit gathering quantum dots and reservoirs connected by tunnel junctions. The effect of time-dependent classical fields on tunneling through quantum dots has been studied since the 1990's ${ }^{76.77}$. Mesoscopic Circuit QED offers the opportunity to study the interaction between microwave photons and quantum dot circuits from a different perspective. Quantum dot circuits are often described in terms of a tunneling model in which the dots and reservoirs contain quasi-localized orbitals coupled by tunnel elements ${ }^{78}$. This decomposition is instrumental in order to particularize the treatment of the reservoirs and account for the irreversibility of transport processes or other reservoir-induced damping effects. Importantly, the tunnel couplings arise from an overlap between neighboring orbitals. This means that these orbitals cannot be considered as orthogonal, except in the limit of weak tunnel couplings (see for instance ${ }^{79}$ ). However, in practice, it may be necessary to take into account a strong inter-dot tunneling. For example, in the case of a DQD, there can exist a strong splitting between bonding and antibonding states, which can be resonant with the cavity $11-15,17$. Depending on the absolute energy scale of the DQD confinement potential, it might be necessary to go to the limit of a non-perturbative inter-dot tunnel coupling to obtain such a splitting. In order to circumvent the difficulty related to the non-orthogonality of the nanocircuit orbitals, we divide the nanocircuit into the ensemble $\mathcal{D}$ of tunnel-coupled quantum dots from one side, and the different reservoirs with an index $R_{p}$ with $p \in\{1, \ldots, N\}$, from the other side. We use an index $o \in\left\{\mathcal{D}, R_{1}, \ldots, R_{N}\right\}$ to denote these different elements, and an index $j$ to denote the different orbitals in a given element $o$. We assume that the low energy spectrum for the whole ensemble $\mathcal{D}$ of the quantum dots can be determined in the absence of the reservoirs as the discrete spectrum of a potential profile with one or several potential wells. This procedure can be performed for an arbitrary inter-dot tunneling strength and leads to exactly orthogonal orbitals, so that $\left\{\hat{c}_{\mathcal{D} j}^{\dagger}, \hat{c}_{\mathcal{D} j^{\prime}}\right\}=\delta_{j, j^{\prime}}$. One can also define exactly orthogonal orbitals in the isolated reservoir $R_{p}$, with $p \in\{1, \ldots, N\}$, so that $\left\{\hat{c}_{R_{p j}}^{\dagger}, \hat{c}_{R_{p^{\prime} j^{\prime}}}\right\}=\delta_{p, p^{\prime}} \delta_{j, j^{\prime}}$. In a second step, one can evaluate the tunnel coupling $t_{\mathcal{D} j, R_{p} j^{\prime}}$ between the orbitals $j$ and $j^{\prime}$ of $\mathcal{D}$ and $R_{p}$ from the overlap between their wavefunctions. This gives $\left\{\hat{c}_{\mathcal{D} j}^{\dagger}, \hat{c}_{R_{p} j^{\prime}}\right\}=0$ only at lowest order in tunneling 80 .
However, using a weak tunneling between $\mathcal{D}$ and $R_{p}$ is not a severe restriction since the large density of states in the reservoirs can compensate for the smallness of the tunnel coupling elements, and lead to a large tunneling rate compatible with the Kondo effect, for instance. To summarize, in the absence of the cavity, the tunnel Hamiltonian writes

$$
\hat{H}_{e}=\sum_{o, j} \varepsilon_{o j} \hat{c}_{o j}^{\dagger} \hat{c}_{o j}+\sum_{o \neq o^{\prime}, j, j^{\prime}}\left(t_{o j, o^{\prime} j^{\prime}} \hat{c}_{o^{\prime} j^{\prime}}^{\dagger} \hat{c}_{o j}+H . c .\right)
$$

where the creation operator $\hat{c}_{o j}^{\dagger}$ corresponds to an orbital $j$ with energy $\varepsilon_{o j}$ and wavefunction $\varphi_{o j}(\vec{r})$ mainly localized inside element $o \in\left\{\mathcal{D}, R_{1}, \ldots, R_{N}\right\}$. One can use $\left\{\hat{c}_{o j}^{\dagger}, \hat{c}_{o^{\prime} j^{\prime}}\right\}=\delta_{o, o^{\prime}} \delta_{j, j^{\prime}}$ at lowest order in the dot/reservoir tunnel couplings.

We now reexpress the photonic pseudo-potential scheme of the previous section in the framework of the tunneling model. For this purpose, one needs to decompose the field operator $\hat{\psi}^{\dagger}(\vec{r})$ associated to tunneling charges on the nanocircuit orbital states. At lowest order in the dot/reservoir tunneling, one can use $\underline{80}$

$$
\hat{\psi}^{\dagger}(\vec{r})=\sum_{o, j} \varphi_{o j}^{*}(\vec{r}) \hat{c}_{o j}^{\dagger} .
$$

Hence, Hamiltonian (8) directly gives

$$
\hat{H}_{\text {tot }}^{\text {tun }}=\hat{H}_{e}+\hat{H}_{\text {Coul }}^{\text {tun }}+\hat{h}_{\text {int }}\left(\hat{a}+\hat{a}^{\dagger}\right)+\hbar \omega_{0} \hat{a}^{\dagger} \hat{a}
$$

with

$$
\hat{h}_{i n t}=\sum_{o, j} \alpha_{o j} \hat{c}_{o j}^{\dagger} \hat{c}_{o j}+\sum_{o j \neq o^{\prime} j^{\prime}}\left(\gamma_{o j, o^{\prime} j^{\prime}} \hat{c}_{o^{\prime} j^{\prime}}^{\dagger} \hat{c}_{o j}+H . c .\right) .
$$

Note that in principle, the term in $\hat{\mathcal{V}}^{2}$ from Eq. (8) renormalizes $\varepsilon_{o j}, t_{o j, o^{\prime} j^{\prime}}$ and the Coulomb interaction term $\hat{H}_{\text {Coul }}^{\text {tun }}$, but this is not essential for the physics we discuss below.

Since the photonic pseudo-potential modifies the potential landscape seen by the tunneling charges, it can naturally affect all the parameters in the tunneling model. First, cavity photons shift the orbital energy $\varepsilon_{o j}$, with a coupling coefficient

$$
\alpha_{o j}=-e \int d r^{3}\left|\varphi_{o j}(\vec{r})\right|^{2} V_{\perp}(\vec{r}) .
$$

In general, $\alpha_{o j}$ strongly depends on the indexes $o$ and $j$ due to the space dependence of $V_{\perp}(\vec{r})$. This makes the cavity-induced orbital energy shifts particularly relevant experimentally. For a standard metallic reservoir, it is reasonable to disregard the dependence of $\alpha_{o j}$ on the orbital index $j$, i.e. $\alpha_{o j} \simeq \alpha_{o}$, because the properties of the electronic wavefunctions in the reservoir can be considered as constant near the Fermi energy $\stackrel{81}{ }$. In this framework, a behavior similar to the capacitive coupling of the cavity central conductor to the reservoirs is recovered. This type of reservoir/cavity coupling enables one to interpret data obtained for a quantum dot 
coupled to two normal metal leads 9,10 . In contrast, $\alpha_{o j}$ may strongly depend on $j$ for $o=\mathcal{D}$, as illustrated for instance by the example of a DQD with an asymmetric $V_{\perp}(\vec{r}) 11,13-15,17-19$.

The Hamiltonian $\hat{H}_{\text {orb }}$ also contains coupling terms in

$$
\gamma_{o j, o^{\prime} j^{\prime}}=-e \int d r^{3} \varphi_{o j}^{*}(\vec{r}) \varphi_{o^{\prime} j^{\prime}}(\vec{r}) V_{\perp}(\vec{r})
$$

with $o j \neq o^{\prime} j^{\prime}$. These terms include photon-induced tunneling terms in $\gamma_{o j, o^{\prime} j^{\prime}}$, with $o \neq o^{\prime}$, and photon-induced transitions internal to $o$, in $\gamma_{o j, o j^{\prime}}$. In principle, all the above cavity/nanocircuit coupling terms can coexist. In practice, we expect the terms in $\alpha_{o j}$ to be dominant over the tunnel terms in $\gamma_{o j, o^{\prime} j^{\prime}}$ with $o \neq o^{\prime}$, which involve a weak overlap between wavefunctions. Nevertheless, as shown in the next section, the signatures of the photon-induced tunneling terms can be boosted by the large number of states they affect in the reservoirs. If $V_{\perp}(\vec{r})$ varies slowly inside $\mathcal{D}$, the photon-induced transitions internal to $\mathcal{D}$ are negligible, i.e. $\gamma_{\mathcal{D} j, \mathcal{D} j^{\prime}} \simeq 0$, since $\left|\varphi_{\mathcal{D} j}\right\rangle$ and $\left|\varphi_{\mathcal{D} j^{\prime}}\right\rangle$ are orthogonal by definition. However, these transitions become possible if the cavity field is weakly screened $\left(V_{\perp}(\vec{r}) \simeq \vec{E}_{0} \cdot \vec{r}\left(\hat{a}+\hat{a}^{\dagger}\right)\right)$, or if a protrusion of a cavity conductor is placed close to one of the quantum dots in order to reinforce the spatial variations of $V_{\perp}(\vec{r})^{11,13,14,17}$. Transitions in $\gamma_{\mathcal{D} j, \mathcal{D} j^{\prime}}$ can be particularly interesting in case of real or artificial spin orbit coupling which mixes spin and orbital states. In this case, the $\gamma_{\mathcal{D} j, \mathcal{D} j^{\prime}}$ terms can correspond to spin-transitions inside a single quantum $\operatorname{dot}^{24-26}$ or a $\mathrm{DQD}^{27-29}$. In the reservoirs, the $\gamma_{R_{p} j, R_{p} j^{\prime}}$ terms are probably always nonresonant due to strong energy relaxation. Hence, we will assume that these terms do not need to be treated explicitly because they can be included in a renormalization of the states $\left|\varphi_{R_{p} j}\right\rangle$.

In practice, the potential $V_{\perp}(\vec{r})$ can be evaluated numerically by removing all nanoconductors from the device and simulating the microwave electromagnetic field in the cavity in the presence of the metallic gates, sources and drains of the nanocircuit. This can be done using standard microwave simulation tools, which can take into account imperfect metals. It enables a realistic evaluation of the elements $\alpha_{o j}$ and $\gamma_{o j, o^{\prime} j^{\prime}}$. Even if $V_{\perp}(\vec{r})$ is not calculated numerically but replaced by a phenomenological expression, the expressions (14) and (15) remain interesting because they set constraints between the different $\alpha_{o j}$ and $\gamma_{o j, o^{\prime} j^{\prime}}$, which all depend on the same $V_{\perp}(\vec{r})$ profile.

We do not give details on the Coulomb interaction term $\hat{H}_{\text {Coul }}^{\text {tun }}$ which stems directly from Eq.(8). If Coulomb blockade in the nanocircuit is already strong in the absence of the cavity, the effect of the cavity on interactions may be disregarded or treated perturbatively. In the next section, we discuss another situation where we assume that the effects of $\hat{H}_{\text {Coul }}^{\text {tun }}$ are negligible due to the large tunnel rate between a quantum dot and a normal metal reservoir. In the general case, Coulomb interactions between the tunneling charges can lead to a large variety of effects, which we will not discuss in this work.
To conclude, we obtain a decomposition of the system Hamiltonian in terms of nanocircuit elements connected by tunnel couplings. While this is reminiscent of the coarse graining description of superconducting microcircuits frequently used in Circuit QED, a full analogy is not possible due to the presence of the orbital degree of freedom in the Mesoscopic QED case. Due to this orbital degree of freedom, we obtain a large variety of electron/photon coupling terms. In this section, we have discussed the case of quantum dot circuits which raises most experimental efforts so far. However, the above approach can be used for any other type of system in which a decomposition in terms of a tunneling model is relevant. This includes quantum point contacts, molecular circuits, metallic tunnel junctions, and hybrid superconducting systems enclosing Andreev bound states or Majorana bound states, for instance. This last case is discussed in more details in section $\coprod \mathrm{II}$.

\section{Discussion on the theoretical context}

Many theoretical works on Mesoscopic QED use as a starting basis a tunnel Hamiltonian where all parameters (orbital energies, tunnel rates,...) are perturbed by corrections proportional to the cavity electric field. These empirical approaches lack of a formal justification and can lead to unphysical predictions. Our work provides a rigorous framework for the use of tunnelling models with a linear light/matter interaction (the superconducting case will be discussed explicitly in section (IE).

As already mentioned above, our approach proceeds along a spirit similar to the PZW transformation, but with modifications necessary to take into account the specificities of Mesoscopic QED, among which a strong space dependence of the cavity electric field on the scale of a nanocircuit, and boundary conditions provided by cavity conductors and voltage-biased DC electrostatic gates. Interestingly, the effect of boundary conditions provided by grounded conductors on the PZW transformation has been discussed recently ${ }^{82}$. However, this Ref. considers only neutral atomic systems affected by locally constant cavity fields (dipolar coupling limit).

\section{E. Mesoscopic QED with superconducting nanocircuits}

\section{Minimal coupling scheme with superconducting nanocircuits}

In the above sections, superconductivity was not explicitly taken into account. It is important to discuss the generalization of our approach to the case of superconducting hybrid nanocircuits, considering the present interest for Majorana fermions 83 or Andreev bound states for instance 84 . For simplicity, we consider below standard BCS superconducting correlations characterized in 
the mean field approach by a superconducting gap $\Delta(\vec{r})$ coupling electrons and holes with opposite spins. The gauge invariant minimal coupling Hamiltonian of Eq.(1) can be generalized as

$$
\hat{H}_{t o t}^{S}=\hat{H}_{t o t}+\int d^{3} r\left(\Delta(\vec{r}) e^{2 \hat{\Phi}(\vec{r})} \hat{\psi}_{\uparrow}^{\dagger}(\vec{r}) \hat{\psi}_{\downarrow}^{\dagger}(\vec{r})+\text { H.c. }\right)
$$

with $\hat{\psi}_{\sigma}^{\dagger}(\vec{r})$ the field operator for tunneling electrons with spin $\sigma \in\{\uparrow, \downarrow\}$ and

$$
\hat{\Phi}(\vec{r})=e\left(\hat{a}-\hat{a}^{\dagger}\right) V_{\perp}(\vec{r}) / \hbar \omega_{0} .
$$

The term $\hat{H}_{t o t}$ is the straightforward generalization of Eq.(11) to the spin dependent case, i.e. the single electron term $\hat{h}_{\mathcal{T}}(\vec{r})$ can have a structure in spin-space, due to magnetic fields and/or spin-orbit coupling, for instance. Hence, $\hat{\psi}(\vec{r})$ must be replaced by ${ }^{t}\left[\hat{\psi}_{\uparrow}(\vec{r}), \hat{\psi}_{\downarrow}(\vec{r})\right]$ in the first term of Eq.(1) and by $\hat{\psi}_{\uparrow}(\vec{r})+\hat{\psi}_{\downarrow}(\vec{r})$ in Eq.(3). Due to gauge invariance, $\hat{h}_{\mathcal{T}}(\vec{r})$ can involve the electron momentum through $-i \hbar \vec{\nabla} \vec{r}+e \vec{A}(\vec{r})$ only. Importantly, we define $\Delta(\vec{r})$ as a gauge invariant quantity, so that the phase parameter $\hat{\Phi}$ ensures the gauge invariance the pairing term of $\hat{H}_{t o t}^{S}$. The form used above for $\hat{\Phi}$ is valid provided photon-induced magnetic effects can be disregarded, in agreement with the approach of section

\section{Photonic pseudo-potential scheme with superconducting nanocircuits}

Using Eqs.(6) and (77) with $\hat{\psi}^{\dagger}(\vec{r}) \hat{\psi}(\vec{r})$ replaced by $\Sigma_{\sigma} \hat{\psi}_{\sigma}^{\dagger}(\vec{r})_{\sigma} \hat{\psi}(\vec{r})$, Hamiltonian (16) is transformed into

$$
\widetilde{\hat{H}_{\text {tot }}^{S}}=\widetilde{\hat{H}_{t o t}}+\int d^{3} r\left(\Delta(\vec{r}) \hat{\psi}_{\uparrow}^{\dagger}(\vec{r}) \hat{\psi}_{\downarrow}^{\dagger}(\vec{r})+\text { H.c. }\right)
$$

where $\widetilde{\hat{H}_{t o t}}$ is again the generalization of Eq. (8) to the spin dependent case. Importantly, Eq.(18) shows that in the photonic pseudo-potential scheme, the phase of the gap term is not affected by photons anymore. This represents one more advantage of this scheme. This result remains valid in the case of $d$-wave or p-wave superconducting correlations 85 .

\section{Mesoscopic QED with Bogoliubov-De Gennes equations}

In the case where electron-electron interactions can be disregarded, Bogoliubov-De Gennes equations are a widely used approach $\underline{86}$, which enables a diagonalization of the circuit Hamiltonian in terms of quasiparticles with creation operator $c_{n}^{\dagger}$. One can define quasiparticles modes in the absence of the cavity, by using

$$
c_{n}^{\dagger}=\int d^{3} r\left[\hat{\psi}_{\uparrow}^{\dagger}(\vec{r}), \hat{\psi}_{\downarrow}^{\dagger}(\vec{r}), \hat{\psi}_{\uparrow}(\vec{r}), \hat{\psi}_{\downarrow}(\vec{r})\right] \cdot w_{n}(\vec{r})
$$

with a spinorial wavefunction

$$
w_{n}(z)={ }^{t}\left[u_{\uparrow}^{n}(z), u_{\downarrow}^{n}(z), v_{\downarrow}^{n}(z),-v_{\uparrow}^{n}(z)\right]
$$

fulfilling

$$
h_{e f f}(\vec{r}) x_{n}(\vec{r})=E_{n} x_{n}(\vec{r})
$$

with $x_{n}(z)={ }^{t}\left[u_{\uparrow}^{n}(z), u_{\downarrow}^{n}(z),-v_{\uparrow}^{n}(z),-v_{\downarrow}^{n}(z)\right]$ and

$$
h_{e f f}(\vec{r})=\left[\begin{array}{cc}
\widetilde{h}_{\mathcal{T}}(\vec{r}) & \Delta(\vec{r}) \sigma_{0} \\
\Delta^{*}(\vec{r}) \sigma_{0} & -\sigma_{y} \widetilde{h}_{\mathcal{T}}^{*}(\vec{r}) \sigma_{y}
\end{array}\right] .
$$

Above, $\sigma_{0}$ and $\sigma_{y}$ are the identity and the second Pauli matrices in spin space. From the definition of $c_{n}^{\dagger}$, one gets

$$
\begin{aligned}
\widetilde{\hat{H}_{\text {tot }}^{S}} & =\sum_{n} E_{n} c_{n}^{\dagger} c_{n}+\hbar \omega_{0} \hat{a}^{\dagger} \hat{a} \\
& +\sum_{n, n^{\prime}}\left(\mathcal{D}_{n n^{\prime}}^{(1)} c_{n}^{\dagger} c_{n^{\prime}}+\mathcal{D}_{n n^{\prime}}^{(2)}\right)\left(\hat{a}+\hat{a}^{\dagger}\right) \\
& +\sum_{n, n^{\prime}}\left(\mathcal{D}_{n n^{\prime}}^{(3)} c_{n}^{\dagger} c_{n^{\prime}}^{\dagger}+\text { H.c. }\right)\left(\hat{a}+\hat{a}^{\dagger}\right)
\end{aligned}
$$

with

$$
\begin{gathered}
\mathcal{D}_{n n^{\prime}}^{(1)}=-e \int d^{3} r w_{n}^{\dagger}(\vec{r}) V_{\perp}(\vec{r}) \tau_{z} w_{n^{\prime}}(\vec{r}), \\
\mathcal{D}_{n n^{\prime}}^{(2)}=-e \int d^{3} r V_{\perp}(\vec{r})\left(\left|v_{\downarrow}^{n}(z)\right|^{2}+\left|v_{\uparrow}^{n}(z)\right|^{2}\right), \\
\mathcal{D}_{n n^{\prime}}^{(3)}=-e \int d^{3} r w_{n}^{\dagger}(\vec{r}) V_{\perp}(\vec{r})\left(\tau_{x}+i \tau_{y}\right) w_{n^{\prime}}^{*}(\vec{r}) / 2
\end{gathered}
$$

and $\tau_{x}, \tau_{y}, \tau_{z}$ Pauli matrices in Nambu space.

\section{Mesoscopic QED with Majorana fermions}

In this section, we discuss the case of a nanocircuit enclosing Majorana bound states, coupled to a cavity. In principle, these self-adjoint bound states can appear at the interface between the topological and non-topological superconducting phases of a nanostructure $\underline{87}-\underline{-89}$. Although an indirect Majorana bound state/cavity coupling mediated by a superconducting quantum bit or a Josephson junction has been considered in many works $\underline{44}-\underline{50}$, a direct coupling can also exist $\underline{56}$. Using our formalism, we can provide a general Hamiltonian describing such a situation. The direct Majorana bound state/cavity coupling occurs due an overlap between neighboring Majorana bound states, caused by the finite size of the nanocircuit. This overlap is naturally dependent on the photonic pseudo-potential. Proposals to obtain Majorana bound states in condensed matter systems rely on the use of superconducting elements, which calls for the use of the results of section IIE For simplicity, we disregard Coulomb interactions and use the framework of 
the Bogoliubov-De Gennes equations of section IIE3. To remain general, we do not specify the details of the system. We consider an ensemble of Majorana bound states with creation operators $C_{M, n}^{\dagger}=C_{M, n}$ and wavefunction $\varphi_{M, n}(\vec{r})$, localized inside a nanoconductor. In general, the nanoconductor also encloses a continuum of ordinary fermionic states $C_{S, n}^{\dagger}$ with wavefunction $\varphi_{S, n}(\vec{r})$ located above an energy gap. We assume that the nanoconductor is tunnel coupled to a fermionic reservoir with orbital states $C_{R, n}^{\dagger}$ with wavefunctions $\varphi_{R, n}(\vec{r})$. Such a reservoir can be used to measure the conductance through the Majorana system ${ }^{83}$. In the limit of weak tunneling, one can reexpress Eq.(22) by using the ensemble of the operators $C_{o, n}^{\dagger}$ with $o \in\{M, R, S\}$, following similar considerations as for the tunneling model of section IIC. This gives:

$$
\begin{aligned}
\widetilde{\hat{H}_{\text {tot }}^{S}} & =\sum_{n<n^{\prime}} 2 i \theta_{n, n^{\prime}} C_{M, n} C_{M, n^{\prime}}+\sum_{o \in\{R, S\}, n} \varepsilon_{n}^{o} C_{o, n}^{\dagger} C_{o, n} \\
& +\sum_{n, n^{\prime}} C_{M, n}\left(t_{n, n^{\prime}}^{M R} C_{R, n^{\prime}}^{\dagger}-\left(t_{n, n^{\prime}}^{M R}\right)^{*} C_{R, n^{\prime}}\right)+\hbar \omega_{0} \hat{a}^{\dagger} \hat{a} \\
& +\sum_{o, o^{\prime} \in\{M, S, R\}}\left(\gamma_{o n, o^{\prime} n^{\prime}}^{(1)} C_{o, n}^{\dagger} C_{o, n^{\prime}}+\gamma_{o n, o^{\prime} n^{\prime}}^{(2)}\right)\left(\hat{a}^{\dagger}+\hat{a}\right) \\
& +\sum_{o, o^{\prime} \in\{M, S, R\}}\left(\gamma_{o n, o^{\prime} n^{\prime}}^{(3)} C_{o, n}^{\dagger} C_{o, n^{\prime}}^{\dagger}+H . c .\right)\left(\hat{a}^{\dagger}+\hat{a}\right) .
\end{aligned}
$$

Above, the coefficients $\gamma_{o n, o^{\prime} n^{\prime}}^{(i)}$ have expressions similar to the $\mathcal{D}_{n n^{\prime}}^{(i)}$ coefficients of Eqs. (23] 25) with $w_{n\left(n^{\prime}\right)}(\vec{r})$ replaced by $\varphi_{o\left(o^{\prime}\right), n\left(n^{\prime}\right)}(\vec{r})$. We use energies $\varepsilon_{n}^{o}$ for the orbitals in the reservoir $(o=R)$ and the nanoconductor continuum of states $(o=S)$, and a tunnel coupling parameter $\theta_{n, n^{\prime}}$ between overlapping Majorana bound states. The Majorana bound states and the reservoir are connected by tunnel coupling terms in $t_{n, n^{\prime}}^{M R}$. For simplicity, we have omitted the DC tunnel coupling between the reservoir and the nanoconductor continuum of states. Photons can shift all the above mentioned parameters, due to the two last lines of Eq. (26). In particular, photons can modify the tunnel coupling between neighboring Majorana bound states, due to the terms in $\gamma_{M n, M n^{\prime}}^{(i)}$, as discussed in Ref.56. Note that in Hamiltonian (26), there is no DC coupling between the Majorana bound states and the nanoconductor continuum of states, which are assumed to be orthogonal by construction of $C_{M, n}$ and $C_{S, n^{\prime}}$. However, there can be photon-induced transitions between the Majorana bound states and the nanocircuit continuum of states due to terms in $\gamma_{M n, S n^{\prime}}^{(i)}$ and $\gamma_{S n^{\prime}, M n}^{(i)}$. Effects related to these last transitions have been discussed in ${ }^{55}$. Our approach provides a general justification for such terms.

\section{EXAMPLE: QUANTUM RC CIRCUIT IN A CAVITY}

\section{A. Hamiltonian of the system}

We now give an example of a specific prediction given by Hamiltonian (12). We consider a cavity coupled to a "quantum RC circuit" made out of a single quantum dot tunnel contacted to a single normal metal reservoir. We assume that the dot has a large intrinsic level spacing so that a single dot orbital with energy $\varepsilon_{d}$ needs to be considered. The dot is capacitively coupled to a DC gate electrode, which enables one to tune $\varepsilon_{d}$. We study the effects of the dot on the photonic mode with frequency $\omega_{0} / 2 \pi$. Following the previous section, the total Hamiltonian of the system is:

$$
\hat{H}_{R C+c a v}=\hat{H}_{e}+\hat{h}_{i n t}\left(\hat{a}+\hat{a}^{\dagger}\right)+\hbar \omega_{0} \hat{a}^{\dagger} \hat{a}+\hat{H}_{b a t h}+\hat{H}_{R F} .
$$

The Hamiltonian

$$
\hat{H}_{e}=\varepsilon_{d} \hat{c}_{d}^{\dagger} \hat{c}_{d}+\int d \varepsilon N_{0} \nu(\varepsilon)\left(\varepsilon \hat{c}^{\dagger}(\varepsilon) \hat{c}(\varepsilon)+t \hat{c}_{d}^{\dagger} \hat{c}(\varepsilon)+t^{*} \hat{c}^{\dagger}(\varepsilon) \hat{c}_{d}\right)
$$

of the dot and its reservoir involves the lead density of states $N_{0} \nu(\varepsilon)$. For later use we have introduced a cutoff $\nu(\varepsilon)=D^{2} /\left(D^{2}+\varepsilon^{2}\right)$. The terms in $t$ describes tunneling between the dot and the reservoir, whose orbitals correspond to fermionic creation operators $\hat{c}_{d}^{\dagger}$ and $c^{\dagger}(\varepsilon)$, respectively, with $\left\{\hat{c}_{d}, \hat{c}_{d}^{\dagger}\right\}=1$ and $\left\{\hat{c}(\varepsilon), \hat{c}^{\dagger}\left(\varepsilon^{\prime}\right)\right\}=$ $\delta\left(\varepsilon-\varepsilon^{\prime}\right) / N_{0} \nu(\varepsilon)$. One can use extra bosonic modes $\hat{b}^{\dagger}(\epsilon)$ with $\left[\hat{b}(\epsilon), \hat{b}^{\dagger}\left(\epsilon^{\prime}\right)\right]=\delta\left(\epsilon-\epsilon^{\prime}\right) / n_{0}$ to describe the intrinsic damping of the cavity, i.e.

$$
\hat{H}_{b a t h}=\int d \epsilon n_{0}\left(\epsilon \hat{b}^{\dagger}(\epsilon) \hat{b}(\epsilon)+\tau \hat{b}^{\dagger}(\epsilon) \hat{a}+\tau^{*} \hat{a}^{\dagger} \hat{b}(\epsilon)\right) .
$$

The coupling between the quantum dot circuit and the cavity is described by the term $\hat{h}_{i n t}\left(\hat{a}+\hat{a}^{\dagger}\right)$ with

$$
\hat{h}_{i n t}=\alpha \hat{c}_{d}^{\dagger} \hat{c}_{d}+\int d \varepsilon N_{0} \nu(\varepsilon)\left(\gamma \hat{c}_{d}^{\dagger} \hat{c}(\varepsilon)+\gamma^{*} \hat{c}^{\dagger}(\varepsilon) \hat{c}_{d}\right) .
$$

It is not necessary to write explicitly the coupling between the chemical potential of the reservoir and the cavity because since there is a single reservoir, it is only the difference between the potentials of the dot and the reservoir which matters. Note that the terminology "quantum RC circuit" mainly refers to the fact that no DC current can flow through the device and dissipation is due to transport through a single tunnel barrier. However, in the general case, the concept of a local capacitor is not sufficient to describe the coupling of the electromagnetic field to the device, since the photonic pseudo potential modifies $\varepsilon_{d}$ and $t$ simultaneously. In practice, one can measure the response of the cavity to a microwave drive with frequency $\omega_{R F} / 2 \pi$, which can be described by

$$
\hat{H}_{R F}=\varepsilon_{R F} e^{i \omega_{R F} t} \hat{a}+\varepsilon_{R F}^{*} e^{-i \omega_{R F} t} \hat{a}^{\dagger} .
$$

Above, we have disregarded Coulomb interactions between the tunneling charges (including the contribution 
from the $\hat{\mathcal{V}}^{2} / \hbar \omega_{0}$ term arising from the unitary transformation of section II.B), because we assume that these interactions are smaller than the tunnel rate $\Gamma=\pi|t|^{2} N_{0}$ between the dot and the reservoir. Such a simplification is frequently performed for interpreting standard current measurements in open quantum dot circuits 92,93 . In practice, one can check the relevance of this hypothesis by measuring for instance the $\mathrm{AC}$ current through the dot versus $\varepsilon_{d}$.
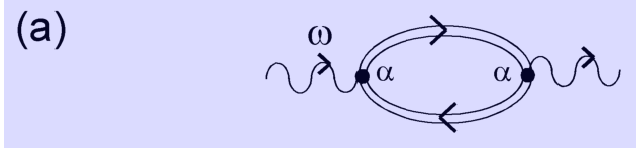

(b)

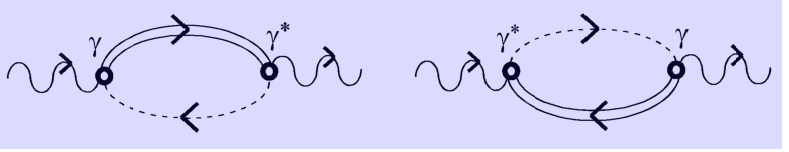

(c)
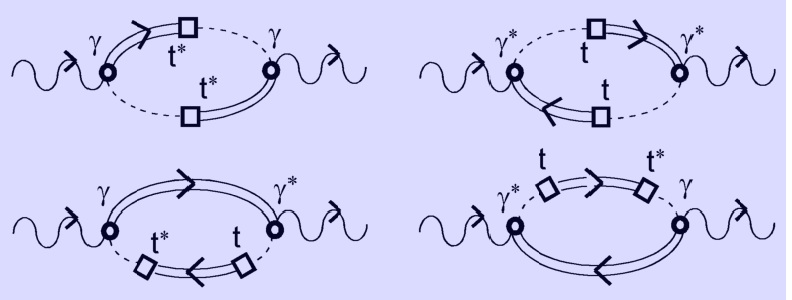

(d)

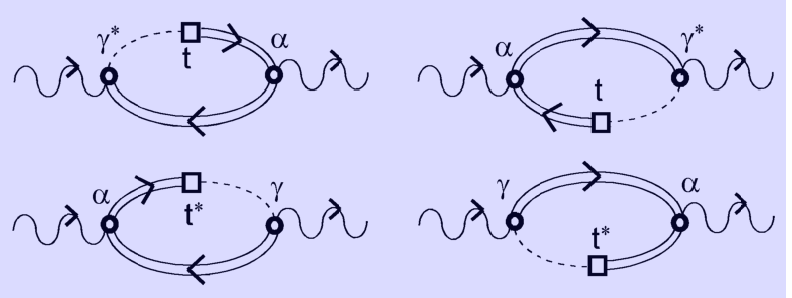

FIG. 2: Scheme of the various contributions to $G_{\hat{h}_{i n t} \hat{h}_{i n t}}(t)$. The wavy lines correspond to photonic propagators, the simple dashed lines to bare electronic propagators in the normal metal reservoir, and the double full lines to electronic propagators in the quantum dot dressed by the dot/reservoir tunneling processes.

\section{B. Cavity frequency pull and damping pull}

Our purpose is to identify signatures of the terms in $\gamma$, which could become visible in the cavity response, considering the recent progress on the Mesoscopic QED technology $y^{90.91}$. In the framework of the linear response theory, the cavity electromagnetic response to $H_{d}$ is determined by the Green's function ${ }^{30} \tilde{G}_{\hat{a}, \hat{a}^{\dagger}}\left(\omega_{R F}\right)$ with $\tilde{G}_{\hat{A}, \hat{B}}(t)=-i \theta(t)\langle[\hat{B}(t), \hat{A}(t=0)]\rangle$ and $\tilde{G}_{\hat{A}, \hat{B}}(\omega)=$ $\int_{-\infty}^{+\infty} d t \tilde{G}_{\hat{A}, \hat{B}}(t) e^{i \omega t}$. One can use the exact relation

$$
\tilde{G}_{\hat{a}, \hat{a}^{\dagger}}(\omega)=\tilde{G}_{0}(\omega)+\hbar^{-2} G_{0}(\omega) \tilde{G}_{\hat{h}_{i n t} \hat{h}_{i n t}}(\omega) G_{0}(\omega)
$$

with $G_{0}(\omega)=\left(\omega-\omega_{0}+i \Lambda_{0}\right)^{-1}$ the free cavity Green's function. In the simplest model where $\tau$ and $n_{0}$ are independent of energy, the constant $\Lambda_{0}=\pi|\tau|^{2} n_{0} / \hbar$ corresponds to pure cavity damping. In the following, we consider the limit $\Lambda_{0} \ll \omega_{0}$ which corresponds to standard cavities. In the limit $\omega_{R F}-\omega_{0}, \Lambda_{0} \ll \Gamma$, one can use a random phase approximation which leads to

$$
\tilde{G}_{\hat{a}, \hat{a}^{\dagger}}\left(\omega_{R F}\right) \simeq\left(\omega_{R F}-\omega_{0}+i \Lambda_{0}-\hbar^{-2} G_{\hat{h}_{i n t} \hat{h}_{i n t}}\left(\omega_{R F}\right)\right)^{-1}
$$

with $G_{\hat{h}_{i n t} \hat{h}_{i n t}}(t)=\left.\tilde{G}_{\hat{h}_{i n t} \hat{h}_{i n t}}(t)\right|_{\hat{h}_{i n t}=0}$ the electronic response function calculated in the absence of the cavity. In the framework of a non-interacting diagrammatic calculation, $G_{\hat{h}_{i n t} \hat{h}_{i n t}}(\omega)$ can be decomposed into the eleven contributions represented in Fig. 2. Our calculation is not perturbative in $t$ since we use an exact expression for the dressed propagator of electrons on the dot, corresponding to the double full lines in Fig.2. There are nine types of diagrams corresponding to the possible ordered pairs formed with the interaction constants $\alpha, \gamma$, and $\gamma^{*}$. The diagrams of Fig.2a correspond to pure contributions from photon-induced orbital shifts in $\alpha^{2}$. The diagrams of Figs. 2b and c correspond to contributions from photon-induced tunneling in $|\gamma|^{2}, \gamma^{2}$ or $\gamma^{* 2}$. Note that the contributions in $|\gamma|^{2}$ have been separated into the diagrams of Fig. 2b which diverge logarithmically for $D$ large and the bottom diagrams in Fig.2c which are regular. There also exist interferences between photoninduced orbital shifts and photon-induced tunneling, as shown by the diagrams of Fig. 2d which depend on $\alpha \gamma$ or $\alpha \gamma^{*}$.

The constants $\Gamma$ and $D$ set the scale of variations of $G_{\hat{h}_{i n t} \hat{h}_{i n t}}(\omega)$. Therefore, in the limit $\omega_{0} \ll$ $\Gamma, D$, one can replace $G_{\hat{h}_{i n t} \hat{h}_{i n t}}\left(\omega_{R F}\right)$ by $G_{\hat{h}_{i n t} \hat{h}_{i n t}}(0)$ in Eq.(33). This gives a cavity frequency pull $\Delta \omega_{0}=$ $G_{\hat{h}_{i n t} \hat{h}_{i n t}}(\omega=0) / \hbar^{2}$ and a cavity damping pull $\Delta \Lambda_{0}=$ $-\left(\omega_{0} / i \hbar^{2}\right) \partial G_{\hat{h}_{i n t} \hat{h}_{i n t}}(\omega) /\left.\partial \omega\right|_{\omega=0}$. In the limit $\Gamma \ll D$ and at zero temperature, one obtains $\Delta \omega_{0}=\Delta \omega_{0}^{a}+\Delta \omega_{0}^{b}$ where

$$
\begin{aligned}
& \Delta \omega_{0}^{a}=\frac{2}{\pi \hbar\left(\Gamma^{2}+\varepsilon_{d}^{2}\right)} \\
& \quad\left(4 \pi^{2} N_{0}^{2} \operatorname{Re}\left[t \gamma^{*}\right]^{2} \Gamma+4 \pi \alpha \operatorname{Re}\left[t \gamma^{*}\right] N_{0} \varepsilon_{d}-\alpha^{2} \Gamma\right)
\end{aligned}
$$

stems from the diagrams of Fig.2,a, c and d, and

$$
\begin{aligned}
\Delta \omega_{0}^{b}= & \frac{2}{\hbar}|\gamma|^{2} N_{0} \frac{D}{D^{2}+\varepsilon_{d}^{2}} \\
& \left(D \log \left[\frac{\Gamma^{2}+\varepsilon_{d}^{2}}{D^{2}}\right]+\left|\varepsilon_{d}\right|\left(2 \arctan \left[\frac{\Gamma}{\left|\varepsilon_{d}\right|}\right]-\pi\right)\right)
\end{aligned}
$$

stems from the diagrams of Fig.2,b. Note that the cutoff $\nu(\varepsilon)$ on the reservoir density of states is necessary to avoid a divergence of $\Delta \omega_{0}^{b}$. The cavity damping pull is 
given by

$$
\Delta \Lambda_{0}=\frac{2 \omega_{0}}{\pi\left(\Gamma^{2}+\varepsilon_{d}^{2}\right)^{2}}\left(\alpha \Gamma-2 \pi \operatorname{Re}\left[t \gamma^{*}\right] N_{0} \varepsilon_{d}\right)^{2} .
$$

Interestingly, $\Delta \omega_{0}^{a}$ and $\Delta \Lambda_{0}$ vanish for $\varepsilon_{d} \gg \Gamma$, whereas $\Delta \omega_{0}^{b}$ vanishes only for $\varepsilon_{d} \gg D$. Above, we have implicitly included the spin degree of freedom in the model. In the spinless case, $\Delta \omega_{0}$ and $\Delta \Lambda_{0}$ should be divided by a factor 2 .

From Eqs. (34 36), at low temperatures, when the cavity has a frequency smaller than the dot/reservoir tunnel rate $\Gamma$ and the reservoir bandwidth $D$, and when $\gamma=0$, the cavity frequency pull and the damping pull fulfill the relation $\Theta=\pi / 2$ with

$$
\Theta=\frac{\alpha^{2} \Delta \Lambda_{0}}{\omega_{0}\left(\hbar \Delta \omega_{0}\right)^{2}} .
$$

This property is a manifestation of the Korringa-Shiba relation ${ }^{94}$, whose universality is still actively discussed for quantum RC circuits ${ }^{60-66}$. Remarkably, we find that this universality is broken for $\gamma$ finite. Hence, one can identify the existence of the $\gamma$ coupling by comparing the $\Delta \omega_{0}\left(\varepsilon_{d}\right)$ and $\sqrt{\Delta \Lambda_{0}}\left(\varepsilon_{d}\right)$ curves (Fig. 3) or studying $\Theta$ (Fig. 4). Here, we present results for $\gamma / \alpha \ll 1$ because from Eqs. (14 15), this is the most probable regime of parameters. When $\gamma=0, \Delta \omega_{0}$ and $\sqrt{\Delta \Lambda_{0}}$ show identical variations with $\varepsilon_{d}$, and are both even functions of $\varepsilon_{d}$, while $\Theta=\pi / 2$. When $\gamma \neq 0, \sqrt{\Delta \Lambda_{0}}$ presents a resonance with $\varepsilon_{d}$ which is wider than that of $\Delta \omega_{0}$ (Fig.3, middle panel). A good resolution on the tails of the $\Delta \omega_{0}$ and $\sqrt{\Delta \Lambda_{0}}$ resonances could also reveal that $\Delta \omega_{0}$ and $\Delta \Lambda_{0}$ are not even with $\varepsilon_{d}$ in the general case, except if $\arg \left[t \gamma^{*}\right]=\pi / 2$ (Fig.3, bottom panel). In principle, even a relatively small $\gamma$ can be detected. Indeed, for $\gamma / \alpha \sim 10^{-4}, \Theta$ already significantly deviates from $\pi / 2$ at $\varepsilon_{d} \sim 0$ where the signals $\Delta \omega_{0}$ and $\sqrt{\Delta \Lambda_{0}}$ are maximal (Fig.4, pink curves). This is because the effect of the photon-induced tunneling term is boosted by the large number of states involved in the reservoirs. To conclude, in order to reveal the photon-induced tunneling terms, one promising possibility is to compare the cavity frequency pull and damping pull caused by a quantum dot with a single normal metal reservoir. Even a relatively weak photon-induced tunneling can affect these quantities because the large number of reservoir states reinforces the tunnel effect.

\section{Experimental state of the art and discussion}

In principle, circuit-QED should enable an accurate determination of $\Theta$ thanks to the use of resonant techniques. The fabrication of a quantum dot with a single normal metal reservoir inside a coplanar cavity is fully accessible with present techniques. However, so far, the closest case studied experimentally is a quantum dot with two normal metal reservoirs, which leads to more complicated physics due to an asymmetric coupling of the two

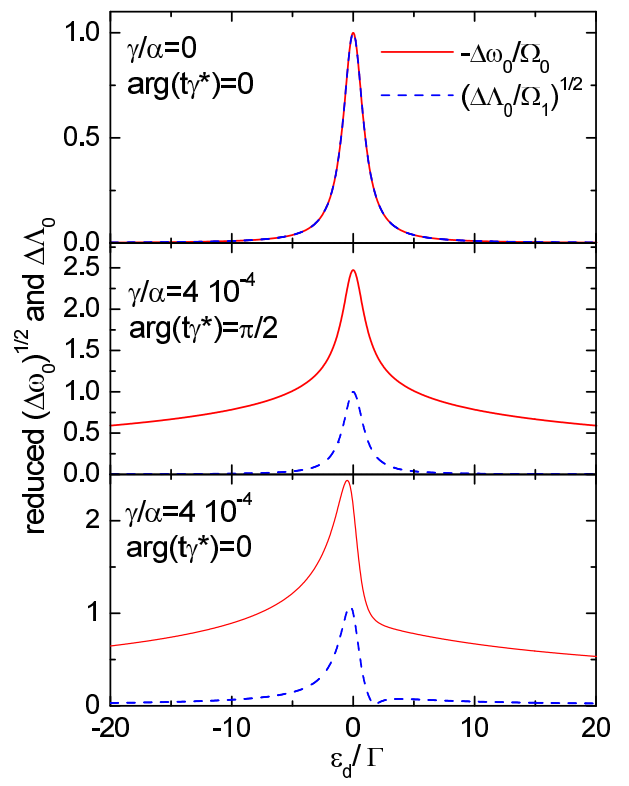

FIG. 3: Reduced $\Delta \omega_{0}$ and $\sqrt{\Delta \Lambda_{0}}$ versus $\varepsilon_{d}$ for different values of $\gamma$. We have used $t / \Gamma=0.001, D=20 \Gamma$, and pulsation scales $\Omega_{0}=2 \alpha^{2} / \pi \Gamma \hbar$ and $\Omega_{1}=\hbar \omega_{0} \Omega_{0} / \Gamma$.

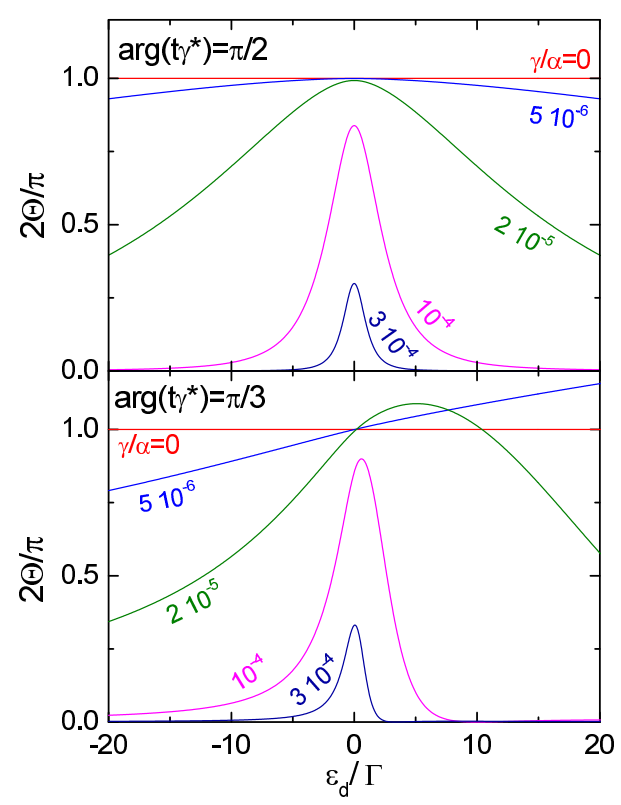

FIG. 4: Ratio $\Theta$ versus $\varepsilon_{d}$ for different values of $\gamma$. We have used the same values of $t, D$ and $\Omega_{0}$ as in Fig.3

reservoirs chemical potentials to the cavity 9.10 . Different types of quantum RC circuits based on 2-DEGs but also carbon nanotubes or semiconducting nanowires deserve an investigation. The ratio $\gamma / \alpha$ should be strongly dependent on the type of nanoconductor and the contacts configuration used.

The physics discussed in this section is related to the problem of a quantum RC circuit which is not coupled to a cavity but directly excited by a classical microwave field 
with amplitude $a_{R F}$ and frequency $\omega_{R F} / 2 \pi$, which corresponds to a term in $\hat{h}_{i n t} a_{R F} \cos \left(\omega_{R F} t\right)$. At low frequencies and $T=0$, it has been predicted ${ }^{60-66}$ that a spinless quantum RC circuit excited classically should have the same admittance as an $\mathrm{RC}$ circuit, with a universal resistance $R_{r e l}=h / 2 e^{2}$. However, this result was obtained in a purely capacitive coupling scheme i.e. $\gamma=0$. Assuming that the admittance of the circuit is determined from the current $\hat{I}=e d \hat{n}_{d} / d t$ with $\hat{n}_{d}=\hat{c}_{d}^{\dagger} \hat{c}_{d}$, this value of $R_{r e l}$ can be recovered by calculating the charge susceptibility $G_{\hat{n}_{d}, \hat{n}_{d}}\left(\omega_{R F}\right)$, i.e. the diagram of Fig. 2.a, which leads to $R_{\text {rel }}=\Theta \hbar / e^{2}=h / 2 e^{2}$ because $\gamma=0$. However, in the general case, one should calculate the current response of the quantum RC circuit from $G_{\hat{n}_{d}, \hat{h}_{i n t}}\left(\omega_{R F}\right)$ because the oscillating drive can also modify the tunnel barrier transparency. Only the diagram of Fig. 2.a and half of the diagrams of Fig. 2d should contribute to $R_{\text {rel }}$, whereas the diagrams of Fig.2.b leading to the logarithmic term of Eq. (35) should not contribute. Therefore, $R_{\text {rel }}$ should show a behavior qualitatively different from $\Theta$. We will not discuss the detailed behavior of $R_{\text {rel }}$ here. We will simply point out that for $\gamma \neq 0, R_{\text {rel }}$ is not universal, and furthermore, $R_{r e l}$ and $\Theta$ are not trivially related. Interestingly, the universal value $R_{\text {rel }}=h / 2 e^{2}$ expected for a spinless system with $\gamma=0$ has been checked experimentally in the limit $T \rightarrow 0$ for a 2 -DEG based circuit ${ }^{93}$. However, this measurement is not incompatible with a finite $\gamma$, due to its uncertainty of the order of $\pm 20 \% 95$.

Note that since $R_{r e l}$ and $\Theta$ are qualitatively different signals, it can be interesting to measure both in the same experiment in order to obtain more information on the system. More generally, the study of tunneling effects can benefit from a simultaneous measurement of the cavity response and the current through the nanocircuit. Such a joint measurement has already been realized in the case of a DQD. The cavity dispersive shift is directly sensitive to the population imbalance between the DQD bounding and antibounding states whereas the current though the DQD corresponds to a more complex combination of state populations and tunnel rates. The simultaneous measurement of the two signals gives stronger constraints to determine the system parameters 17 . Another possibility to gain more information on Mesoscopic QED systems might be to measure cross-correlations between the electronic current and the cavity output field.

Importantly, the example of the quantum RC circuit shows that dissipation of the cavity photons has to be considered carefully, since an open quantum dot circuit with a single contact can already induce photon dissipation. On a more general level, photonic dissipation in Mesoscopic QED is a very rich problem. Another interesting possibility would be to prepare non-trivial quantum photonic states by combining the effects of photonic and electronic dissipation with the non-linearity of quantum dot circuits, by analogy with the methods developped for two level systems 96.97 . This so-called "reservoir engineering" could benefit from the specificities of nanocircuits like for instance the use of reservoirs with specific electronic orders or finite bias electronic transport .

\section{CONCLUSION}

Mesoscopic QED has common ingredients with atomic Cavity QED, like the relevance of an orbital degree of freedom, which does not exist for superconducting qubits. It has also common ingredients with standard Circuit QED, like the tunneling physics and the strong inhomogeneities of the photonic modes. It is therefore necessary to develop a specific description which combines all these ingredients. In the case where photon induced magnetic effects can be disregarded, one can express the coupling between tunneling quasiparticles and photons in terms of a scalar photonic pseudo-potential. In the framework of a tunneling model, this leads to photon-induced orbital energy shifts, which coexist with photon-induced tunneling terms and photon-induced local orbital transitions. To illustrate the richness of our approach, we have discussed the example of a cavity coupled to a quantum RC circuit, i.e. a single quantum dot coupled to a single normal metal reservoir. The photon-induced tunneling terms between the dot and the reservoir induce a non-universal relation between the cavity frequency pull and the cavity damping pull, contrarily to what is expected with purely capacitive coupling schemes at low temperatures ${ }^{60-66}$. This case represents only one example of use for our formalism. We have given explicit Hamiltonians for the cases of a multi-quantum dot circuit in a cavity, and a superconducting nanostructure enclosing Majorana bound states coupled to a cavity. One could also consider cavities coupled to quantum point contacts, molecular circuits, and metallic tunnel junctions, for instance.

We acknowledge useful discussions with M. Baillergeau, L. E. Bruhat, C. Cohen-Tannoudji, M. C. Dartiailh, M. R. Delbecq, M. P. Desjardins, $R . B$. Saptsov, M. Trif, and J. J. Viennot. This work was financed by the ERC Starting grant CirQys, the EU FP7 project SE2ND[271554] and the ANR-NanoQuartet [ANR12BS1000701] (France).

\section{APPENDIX A: EFFECTIVE MODEL FOR MESOSCOPIC QED DEVICES}

\section{A. Definition of the model}

In the main text, Eqs (10)-(4) have been introduced on the basis of a physical discussion and gauge invariance considerations. It is also instructive to use an effective model which separates spatially the tunneling and plasmonic parts of the nanocircuit. Note that this effective model does not aim at calculating quantitatively the nanocircuit/cavity couplings. It rather aims at providing 
(a)

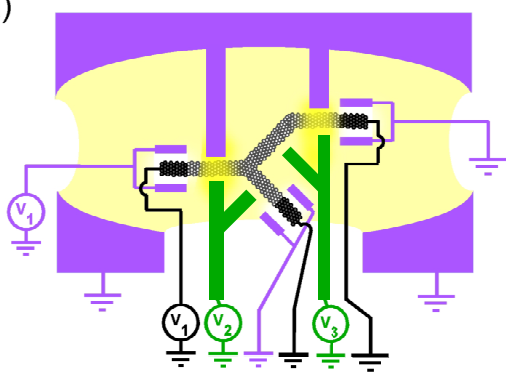

(b)

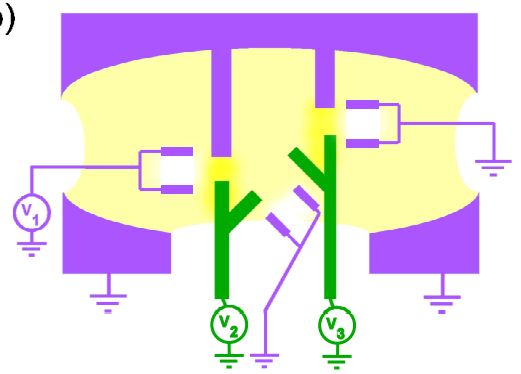

(c)

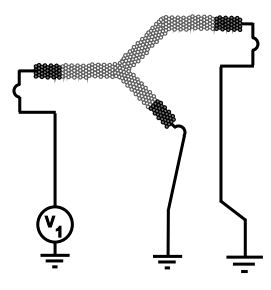

FIG. 5: (a) Effective model for the circuit of Fig.1, where the nanocircuit reservoirs are decomposed into effective orbital reservoirs (dark grey elements) and effective plasmonic reservoirs (nearby purple elements) (b) Ensemble $\mathcal{C}$ of the perfect conductors considered for the generation of the photonic modes. The photonic field in yellow is inhomogeneous, as represented by darker yellow areas near the cavity protusions, and by the white (screening) areas between the small purple conductors (c) Effective orbital nanocircuit $\mathcal{O}$.

a full mathematical justification for the form of Eqs. (1)(44) and a deeper insight on the underlying physics. For simplicity, we assume that the nanoconductors host only individual electronic orbital modes since their electronic density is generally very low. We assume that the plasmonic modes in the fermionic reservoirs (blue elements in Fig. 1) are ultra fast due to the absence of dynamical Coulomb blockade $\mathrm{6s}^{68}$. In this case, one can decompose each fermionic reservoir into a purely orbital conductor (dark grey elements in Fig.5.a) which contains no plasmons and is tunnel coupled to the nanocircuit, and perfect conductors (small purple elements in Fig.5.a) which can host plasmonic modes, but are only capacitively coupled to the nanoconductors. These perfect conductors, or "effective plasmonic reservoirs", can be placed such that the partial or full decrease of the photonic fields around the nanocircuit orbital states is reproduced at least qualitatively. We denote with $\mathcal{C}$ the ensemble of the perfect conductors, which includes the cavity conductors, gate electrodes, and the effective plasmonic reservoirs (see Fig. 5.b). Some of these conductors are voltage biased and some others are left floating, like for instance the central conductor in a coplanar cavity. The rest of the circuit represents an effective orbital nanocircuit $\mathcal{O}$ where tunneling physics prevails (see Fig. 5.c). Note that in principle, to grant current conservation, the "orbital" reservoirs have to be connected to voltage sources through

wirings which host plasmonic modes, but one can assume that they are too far from the nanoconductors to have any significant influence on the nanocircuit/cavity coupling.

For later use, we attribute to each conductor of the ensemble $\mathcal{C}$ an index $i$ and we note $\mathcal{S}_{i}$ and $\mathcal{V}_{i}$ the corresponding surface and volume. In the following treatment we make a distinction between the conductors $i \in \mathbb{F}$ which are left floating with a constant total charge $Q_{i}$, and the conductors $i \in \mathbb{B}$ which are voltage-biased with a constant voltage $V_{i}$ or grounded $\left(V_{i}=0\right)$. The generators imposing the voltages $V_{i}$ can be omitted from the description and included in electromagnetic boundary conditions since they are far from the cavity and nanocircuits. The volume $\mathcal{V}_{\mathcal{O}}$ complementary to $\sum_{i} \mathcal{V}_{i}$ hosts a charge distribution $\rho(\vec{r}, t)=\sum_{\alpha \in \mathcal{O}} e_{\alpha} \delta\left(\vec{r}-\vec{q}_{\alpha}\right)$ of particles $\alpha$ with charges $e_{\alpha}$ at positions $\vec{q}_{\alpha}$, which belong to the nanocircuit $\mathcal{O}$. We also define the corresponding current distribution $\vec{j}(\vec{r}, t)=\sum_{\alpha \in \mathcal{O}} e_{\alpha} \overrightarrow{\dot{q}} \alpha \delta\left(\vec{r}-\vec{q}_{\alpha}\right)$.

\section{B. Decomposition of fields in harmonic, longitudinal, and transverse components.}

In Cavity QED, the treatment of the boundary conditions provided by the cavity conductors is usually omitted, because the atoms are very far from the cavity mirrors. Therefore, a procedure based on a spatial Fourier transformation can be used to separate the transverse electric field $\vec{E}_{\perp}$, which has no divergence, from the longitudinal electric field $\vec{E}_{\|}$, which has no rotational $l^{4}$. It is found that the value of $\vec{E}_{\|}$is instantaneously imposed by the charge distribution $\rho(\vec{r}, t)$ associated to the atomic system. In contrast, the transverse fields $\vec{E}_{\perp}$ and $\vec{B}=\vec{B}_{\perp}$ correspond to propagating modes. In Mesoscopic QED, it is necessary generalize this procedure to account for the presence of the perfect conductors of the ensemble $\mathcal{C}$, including voltage-biased electrostatic gates. As already mentioned in the previous section, we use a charge distribution $\rho(\vec{r}, t)$ which includes the charges from the $\mathcal{O}$ subsystem only. We will take into account the screening charges on the surface of the conductors of $\mathcal{C}$ through electromagnetic boundary conditions.

Inside $V_{\mathcal{O}}, \rho(\vec{r}, t), \vec{j}(\vec{r}, t)$, and the total electric and magnetic fields $\vec{E}(\vec{r}, t)$ and $\vec{B}(\vec{r}, t)$ are related by Maxwell's equations. To understand how these relations are affected by the screening charges lying on $\mathcal{C}$, we introduce the Hodge decomposition of a generic field $\vec{f}$ as $\vec{f}=\vec{f}_{\|}+\vec{f}_{\perp}+\vec{f}_{\text {harm }}$, where $\vec{f}_{\|}$has a finite divergence but no rotational, $\vec{f}_{\perp}$ has a finite rotational but no divergence, and $\vec{f}_{\text {harm }}$ has none $\underline{69,70}$. A similar decomposition was invoked very recently in the context of Cavity $\mathrm{QED}^{82}$. However, in this Ref., the effects of the DC voltage biases $V_{i}$ or the floating charges $Q_{i}$ are not considered (i.e. $V_{i}=0$ is used for any $i$ ). Besides, the volume $V_{\mathcal{O}}$ outside the cavity conductors is assumed to be bounded. 
This hypothesis is not possible in our case due to openings in the cavity planes, which are necessary to connect the nanocircuits to their outside voltage bias circuitry. Hence, we need to perform the Hodge decomposition in the fully general case. Below, we explain how to perform this task.

It is convenient to introduce a potential $\Phi_{\text {harm }}(\vec{r})$ and a Green's function $G\left(\vec{r}, \vec{r}^{\prime}\right)$ which fulfill the static Laplace equations:

$$
\Delta_{\vec{r}} \Phi_{\text {harm }}(\vec{r})=0
$$

and

$$
\Delta_{\vec{r}} G\left(\vec{r}, \vec{r}^{\prime}\right)=-\delta\left(\vec{r}-\vec{r}^{\prime}\right) / \varepsilon_{0}
$$

for $\left(\vec{r}, \vec{r}^{\prime}\right) \in \mathcal{V}_{\mathcal{O}}^{2}$, with boundary conditions

$$
\begin{array}{r}
\int_{\mathcal{S}_{i}} d^{2} r \vec{\nabla}{ }_{\vec{r}} \Phi_{\text {harm }}(\vec{r}) \cdot \vec{n}=-Q_{i} \text { for } i \in \mathbb{F}, \\
\Phi_{\text {harm }}(\vec{r})=V_{i} \text { for } \vec{r} \in \mathcal{S}_{i} \text { and } i \in \mathbb{B}
\end{array}
$$

and

$$
\begin{aligned}
& \int_{\mathcal{S}_{i}} d^{2} r \vec{\nabla}{ }_{r} G\left(\vec{r}, \vec{r}^{\prime}\right) \cdot \vec{n}=0 \text { for } i \in \mathbb{F}, \\
& G\left(\vec{r}, \vec{r}^{\prime}\right)=0 \text { for } \vec{r} \in \mathcal{S}_{i} \text { and } i \in \mathbb{B} .
\end{aligned}
$$

Above, $\vec{n}$ is the outward pointing unit vector perpendicular to $\mathcal{S}_{i}$. One can check that each of these two sets of Eqs. has a unique solution provided $\mathbb{B}$ is not empty ${ }^{98}$. From Eqs. 38, 43) and the Maxwell Eqs., one can check that:

$$
\begin{gathered}
\vec{E}_{\text {harm }}(\vec{r})=-\vec{\nabla}_{\vec{r}} \Phi_{\text {harm }}(\vec{r}), \\
\vec{E}_{\|}(\vec{r}, t)=-\int d^{3} r^{\prime} \vec{\nabla}_{\vec{r}} G\left(\vec{r}, \vec{r}^{\prime}\right) \rho\left(\vec{r}^{\prime}, t\right)
\end{gathered}
$$

and

$$
\vec{E}_{\perp}(\vec{r}, t)=\vec{E}(\vec{r}, t)-\vec{E}_{\|}(\vec{r}, t)-\vec{E}_{h a r m}(\vec{r})
$$

while $\vec{B}_{\perp}(\vec{r}, t)=\vec{B}(\vec{r}, t)$ is still valid. We also find $\vec{j}=\vec{j}_{\|}+\vec{j}_{\perp}$ with

$$
\vec{j}_{\|}(\vec{r}, t)=-\left(\partial \vec{E}_{\|}(\vec{r}, t) / \partial t\right) / c^{2} \mu_{0}
$$

and

$$
\vec{j}_{\perp}(\vec{r}, t)=\vec{j}(\vec{r}, t)-\vec{j}_{\|}(\vec{r}, t) .
$$

The harmonic component $\vec{E}_{\text {harm }}$ represents a static contribution to the electric field, set by the charges $Q_{i}$ or potentials $V_{i}$ imposed on the conductors $i$. Thanks to the use of $G\left(\vec{r}, \vec{r}^{\prime}\right)$, the definitions (45] 48) take into account the effect of the screening charges lying on $\mathcal{C}$. Using the Maxwell equations and the expressions (44,48), one can check that the longitudinal components $\vec{E}_{\|}$and $\vec{j}_{\|}$ are set instantaneously by $\rho(\vec{r}, t)$ since

$$
\vec{\nabla}_{\vec{r}} \cdot \vec{E}_{\|}(\vec{r}, t)=\rho(\vec{r}, t) / \varepsilon_{0}
$$

for $\vec{r} \in \mathcal{V}_{\mathcal{O}}$. Furthermore, $\vec{B}_{\perp}, \vec{E}_{\perp}$ and $\vec{j}_{\perp}$ follow an independent system of propagation equations, i.e.

$$
\begin{gathered}
\vec{\nabla}_{\vec{r}} \wedge \vec{E}_{\perp}(\vec{r}, t)=-\partial \vec{B}_{\perp}(\vec{r}, t) / \partial t \\
\vec{\nabla}_{\vec{r}} \wedge \vec{B}_{\perp}(\vec{r}, t)=\mu_{0} \vec{j}_{\perp}(\vec{r}, t)+\frac{1}{c^{2}} \frac{\partial}{\partial t} \vec{E}_{\perp}(\vec{r}, t)
\end{gathered}
$$

for $\vec{r} \in \mathcal{V}_{\mathcal{O}}$. The above equations have to be supplemented with the boundary conditions

$$
\int_{\mathcal{S}_{i}} d^{2} r \vec{E}_{\|(\perp)}(\vec{r}, t) \cdot \vec{n}=0 \text { for } i \in \mathbb{F}
$$

$$
\int_{\ell_{i j}} \overrightarrow{d r} \cdot \vec{E}_{\|(\perp)}(\vec{r}, t)=0 \text { for } \vec{r} \in \mathcal{S}_{i} \text { with }(i, j) \in \mathbb{B}^{2}
$$

with $\ell_{i j}$ any trajectory connecting conductors $i$ and $j$.

\section{Mesoscopic QED classical Lagrangian.}

At this stage, it is convenient to introduce scalar and vector potentials $U$ and $\vec{A}$ in the Coulomb gauge i.e. $\vec{\nabla} \vec{r} \cdot \vec{A}(\vec{r}, t)=0$. We also define the decomposition $U(\vec{r}, t)=U_{\|}(\vec{r}, t)+\Phi_{\text {harm }}(\vec{r})$, such that

$$
\begin{aligned}
& \vec{E}_{\|}(\vec{r}, t)=-\vec{\nabla}_{\vec{r}} U_{\|}(\vec{r}, t), \\
& \vec{E}_{\perp}(\vec{r}, t)=-\partial \vec{A}(\vec{r}, t) / \partial t,
\end{aligned}
$$

and

$$
\vec{B}=\vec{\nabla}_{\vec{r}} \wedge \vec{A}(\vec{r}, t) .
$$

A comparison between Eqs. (39,43), and (49,52:53) leads to the identification

$$
U_{\|}(\vec{r}, t)=\int_{\mathcal{V}_{\mathcal{O}}} d^{3} r^{\prime} G\left(\vec{r}, \vec{r}^{\prime}\right) \rho\left(\vec{r}^{\prime}, t\right)
$$

up to a global constant which one can disregard. Hence, $\vec{E}_{\|}$but also $U_{\|}$are instantaneously set by the charge distribution $\rho(\vec{r}, t)$. In contrast, the transverse fields $\vec{E}_{\perp}$ and $\vec{B}$ are determined by $\vec{A}$ which follows the propagation equation

$$
\square_{\vec{r}}(\vec{A}(\vec{r}, t))=-\mu_{0} \vec{j}_{\perp}(\vec{r}, t)
$$

given by Eq.(51), with $\square_{\vec{r}}=\Delta_{\vec{r}}-\left(\partial^{2} / c^{2} \partial t^{2}\right)$, and boundary conditions similar to Eqs.(52)-(53). In this framework, a proper classical Lagrangian is

$$
\begin{aligned}
L & =\frac{1}{2} \sum_{\alpha} m_{\alpha} \dot{q}_{\alpha}^{2}-\frac{1}{2} \sum_{\alpha} e_{\alpha} U_{\|}\left(\vec{q}_{\alpha}, t\right) \\
& -\sum_{\alpha} e_{\alpha} \Phi_{\text {harm }}\left(\vec{q}_{\alpha}\right)+\sum_{\alpha} e_{\alpha} \overrightarrow{\dot{q}}_{\alpha} \cdot \vec{A}\left(\vec{q}_{\alpha}, t\right) \\
& +\frac{\varepsilon_{0}}{2} \int_{\mathcal{V}_{\mathcal{O}}} d^{3} r\left(\left|\frac{d}{d t} \vec{A}(\vec{r}, t)\right|^{2}-c^{2}\left|\vec{\nabla}_{\vec{r}} \wedge \vec{A}(\vec{r}, t)\right|^{2}\right)
\end{aligned}
$$


with $\alpha \in \mathcal{O}$ in the sums. The Lagrangian equations deriving from $L$ correspond to Eq.(58) and to the standard Newton equations for the motion of particles $\alpha$.

\section{Mesoscopic QED Hamiltonian}

From Eq. (59), one can check that $\vec{p}_{\alpha}=m_{\alpha} \vec{q}_{\alpha}+$ $e_{\alpha} \vec{A}\left(\vec{q}_{\alpha}, t\right)$ is the conjugate of $\vec{q}_{\alpha}$ and $\vec{\Pi}_{\perp}(\vec{r}, t)=$ $-\varepsilon_{0} E_{\perp}(\vec{r}, t)$ is the conjugate of $\vec{A}(\vec{r}, t)$. Therefore one can express the Hamiltonian of the system as $H=\sum_{\alpha} \vec{p}_{\alpha} \cdot \vec{q}_{\alpha}+\int_{\mathcal{V}_{\mathcal{O}}} d^{3} r\left(\vec{\Pi}_{\perp}(\vec{r}, t) \cdot \vec{A}(\vec{r}, t)\right)-L$. This Hamiltonian can be quantized straightforwardly by replacing $\vec{p}_{\alpha}, \vec{q}_{\alpha}, \vec{\Pi}_{\text {and }} \vec{A}$ by operators $\overrightarrow{\hat{p}}_{\alpha}, \overrightarrow{\hat{q}}_{\alpha}, \overrightarrow{\hat{\Pi}}$ and $\overrightarrow{\hat{A}}$. This gives

$$
\begin{aligned}
\hat{H} & =\frac{1}{2 m_{\alpha}} \sum_{\alpha}\left(\overrightarrow{\hat{p}}_{\alpha}-e_{\alpha} \overrightarrow{\hat{A}}\left(\overrightarrow{\hat{q}}_{\alpha}\right)\right)^{2} \\
& +\frac{1}{2} \sum_{\alpha} e_{\alpha} U_{\|}\left(\overrightarrow{\hat{q}}_{\alpha}\right)+\sum_{\alpha} e_{\alpha} \Phi_{\text {harm }}\left(\overrightarrow{\hat{q}}_{\alpha}\right) \\
& +\frac{1}{2} \int_{\mathcal{V}_{\mathcal{O}}} d^{3} r\left(\left.\frac{1}{\varepsilon_{0}}\left|\overrightarrow{\hat{\Pi}}^{2}+\frac{1}{\mu_{0}}\right| \vec{\nabla}_{\vec{r}} \wedge \overrightarrow{\hat{A}}(\vec{r})\right|^{2}\right) .
\end{aligned}
$$

In principle, the ensemble of the charges $\alpha \in \mathcal{O}$ includes tunneling electrons $(\alpha \in \mathcal{T})$ but also ions and valence electrons from an underlying crystalline structure. In the main text, the two latter are assumed to be decoupled from the cavity and are thus treated in a mean field approach. Note that the harmonic potential term in the above Hamiltonian is omitted by Ref $\underline{82}$. In our case, this term is crucial to account for the effect of DC electrostatic gates.

Assuming that the coupling between $\mathcal{C}$ and $\mathcal{O}$ is perturbative, the fields $\overrightarrow{\hat{A}}$ and $\overrightarrow{\hat{\Pi}}$ can be expressed in terms of photonic modes calculated in the absence of $O$, using for instance Eq.(58) with $\vec{j}_{\perp}=0$ We treat explicitly only one of these modes in Eq.(4). Screening charges on the nanocircuits reservoirs can strongly modify $\overrightarrow{\hat{A}}$. This is why, in the general case, it is not adequate to make a perturbative treatment between the photonic field generated by the empty cavity and the whole nanocircuit. Instead, the present work considers a perturbative treatment between the photonic field generated by the plasmonic modes in the whole Mesoscopic QED device, and the effective orbital nanocircuit $\mathcal{O}$. Similarly, in superconducting Circuit QED, the cavity modes can be strongly renormalized by the presence of a superconducting quantum bit, and perturbation schemes must therefore be defined carefully ${ }^{99}$.

In this Appendix, the renormalization of the bare cavity modes by the nanocircuit plasmons is described formally by supplementary boundary conditions imposed by effective plasmonic reservoirs. This enables a simple mathematical justification for the form of Eqs (11)-(4). In order to get realistic estimates of the fields $\overrightarrow{\hat{A}}$ and $\vec{\Pi}$, one can make a numerical microwave simulation using the real device geometry, with the nanoconductors omitted (see main text).

\section{APPENDIX B: ADVANTAGES OF THE PHOTONIC PSEUDO-POTENTIAL SCHEME}

The advantages of a formalism without the $\hat{A}^{2}$ terms have already been discussed thoroughly for Cavity QED ${ }^{4}$. However, since the physical ingredients and measured quantities are different in Cavity and Mesoscopic QED, it is useful to discuss more specifically the advantages of the photonic pseudo-potential scheme of section $\amalg E 2$.

The first quantity which is generally measured in Mesoscopic QED experiments is the cavity frequency pull for a given cavity mode, which is modified by the nanocircuits coupled to the cavity. Since the minimal coupling scheme and the photonic pseudo-potential scheme are related by a unitary transformation, they must of course predict the same cavity pull. It is nevertheless instructive to check this equivalence for a simple example. Here, we use a simpler approach than in section IV, because we do not need to particularize the effect of the nanocircuit reservoirs and we will not discuss the cavity damping pull. We consider a cavity coupled to a nanocircuit with unperturbed many electron states $\left|\varphi_{j}\right\rangle$ which satisfy the general equation $\hat{H}_{0}\left|\varphi_{j}\right\rangle=E_{j}\left|\varphi_{j}\right\rangle$ with

$$
\hat{H}_{0}=\sum_{\alpha}\left(\frac{\hat{p}_{\alpha}^{2}}{2 m_{\alpha}}-e \Phi_{t o t}\left(\overrightarrow{\hat{q}}_{\alpha}\right)\right)+\frac{e^{2}}{2} \sum_{\alpha, \alpha^{\prime}} G\left(\overrightarrow{\hat{q}}_{\alpha}, \overrightarrow{\hat{q}}_{\alpha^{\prime}}\right)
$$

and $\Phi_{\text {tot }}\left(\overrightarrow{\hat{q}}_{\alpha}\right)=\Phi_{\text {harm }}\left(\overrightarrow{\hat{q}}_{\alpha}\right)+V_{\text {conf }}\left(\overrightarrow{\hat{q}}_{\alpha}\right)$. We discuss the limit where the nanocircuit and the cavity are not resonant i.e. $E_{j}-E_{j^{\prime}} \neq \hbar \omega_{0}$ for any $j$ and $j^{\prime}$. At second order in the cavity/nanocircuit coupling (i.e. $V_{\perp}$ or $\overrightarrow{\mathcal{A}}$ ), an elementary perturbation theory gives the cavity pull

$$
\Delta \hbar \omega_{0, j}=\sum_{j^{\prime}, \alpha, \alpha^{\prime}} \frac{2\left(\hbar \omega_{0}\right)^{2}\left(E_{j}-E_{j^{\prime}}\right) \mathcal{M}_{j j^{\prime}}^{\alpha \alpha^{\prime}}}{\left(\left(E_{j}-E_{j^{\prime}}\right)^{2}-\left(\hbar \omega_{0}\right)^{2}\right)}
$$

with

$$
\mathcal{M}_{j j^{\prime}}^{\alpha \alpha^{\prime}}=\left\langle\varphi_{j}\left|V_{\perp}\left(\overrightarrow{\hat{q}_{\alpha}}\right)\right| \varphi_{j^{\prime}}\right\rangle\left\langle\varphi_{j^{\prime}}\left|V_{\perp}\left(\overrightarrow{\hat{q}_{\alpha^{\prime}}}\right)\right| \varphi_{j}\right\rangle
$$

Note that $\Delta \hbar \omega_{0, j}$ depends on the state $\left|\varphi_{j}\right\rangle$ occupied by the nanocircuit. This can be used, in principle, for a noninvasive readout of the nanocircuit state in the nonresonant regime ${ }^{2}$. Equation (62) can be obtained by using Hamiltonian (8) or equivalently Hamiltonian (11). In order to check the equivalence between the two approaches, it is necessary to invoke the completeness relation $\sum_{j}\left|\varphi_{j}\right\rangle\left\langle\varphi_{j}\right|=1$ for the nanocircuit states. Interestingly, one can check that the $\hat{A}^{2}$ terms in Eq.(1) give a contribution

$$
\Delta \hbar \omega_{0, j}^{2 p h}=\sum_{j^{\prime}, \alpha, \alpha^{\prime}} 2\left(E_{j}-E_{j^{\prime}}\right) \mathcal{M}_{j j^{\prime}}^{\alpha \alpha^{\prime}}
$$


to $\Delta \hbar \omega_{0, j}$, which is not negligible for $\left|E_{j}-E_{j^{\prime}}\right| \gg \hbar \omega_{0}$. We conclude that in the framework of Mesoscopic QED where the cavity frequency pull is a central quantity, it is particularly important to eliminate in a rigorous way the $\hat{A}^{2}$ terms if one wants to use a linear light/matter coupling. The photonic pseudo-potential scheme completes such a task.

The cavity frequency pull is not the only means to characterize the interactions between a nanocircuit and a cavity. A more general workout of Mesoscopic QED devices requires the knowledge of the matrix elements between the different nanocircuit eigenstates, generated by the cavity photons. Another argument in strong favor of the use of the photonic pseudo-potential scheme is the general relation

$$
\begin{aligned}
\left\langle\varphi_{j}\left|V_{\perp}\left(\overrightarrow{\hat{q}}_{\alpha}\right)\right| \varphi_{j^{\prime}}\right\rangle & =\frac{i e}{2 m_{\alpha}\left(E_{j^{\prime}}-E_{j}\right)} \\
& \left\langle\varphi_{j}\left|\overrightarrow{\hat{p}}_{\alpha} \cdot \overrightarrow{\mathcal{A}}\left(\overrightarrow{\hat{q}}_{\alpha}\right)+\overrightarrow{\mathcal{A}}\left(\overrightarrow{\hat{q}}_{\alpha}\right) \cdot \overrightarrow{\hat{p}}_{\alpha}\right| \varphi_{j^{\prime}}\right\rangle .
\end{aligned}
$$

It indicates that the single-photon coupling elements decay more quickly with $\left(E_{j^{\prime}}-E_{j}\right)$ in the photonic pseudopotential scheme. In practice one often uses a truncated space for the nanocircuit electronic states which is difficult to handle or determine globally. This is what we do for instance in section IV, where we consider a single orbital quantum dot. Equation (65) shows that in this case it is more accurate to use Eq.(8) to predict the cavity behavior.

Finally, the photonic pseudo-potential scheme involves only photon-independent superconducting gap terms (see section \E). This can be another significant advantage considering the already rich structure of the nanocircuit/light coupling in the absence of superconductivity.
1 J.M. Raimond, M Brune., and S. Haroche, Manipulating quantum entanglement with atoms and photons in a cavity, Rev. Mod. Phys. 73, 565 (2001).

2 A. Wallraff, et al. Strong coupling of a single photon to a superconducting qubit using circuit quantum electrodynamics, Nature 431, 162 (2004).

${ }^{3}$ H. Paik et al., Observation of High Coherence in Josephson Junction Qubits Measured in a Three-Dimensional Circuit QED Architecture, Phys. Rev. Lett. 107, 240501 (2011).

4 C. Cohen-Tannoudji, J. Dupont-Roc, and G. Grynberg, Photons and Atoms: Introduction to Quantum Electrodynamics (New York: Wiley) 1997.

5 M.H. Devoret, D. Esteve, C. Urbina, Single electron phenomena in metallic nanostructures, p. 605 in Mesoscopic Quantum Physics / Les Houches (Elsevier, Amsterdam, Netherlands, 1995).

${ }^{6}$ V. Bouchiat, D. Vion, P. Joyez, D. Esteve, and M. H. Devoret, Quantum coherence with a single Cooper pair, Physica Scripta T76, 165-170 (1998).

7 Y. Nakamura, Yu. A. Pashkin, and J. S. Tsai, Coherent control of macroscopic quantum states in a single-Cooperpair box, Nature 398, 786 (1999).

8 A. Blais, R.-S. Huang, A. Wallraff, S. M. Girvin, and R. J. Schoelkopf, Cavity quantum electrodynamics for superconducting electrical circuits: An architecture for quantum computation, Physical Review A 69, 062320 (2004).

9 M.R. Delbecq, et al. Coupling a Quantum Dot, Fermionic Leads, and a Microwave Cavity on a Chip, Phys. Rev. Lett. 107, 256804 (2011)

10 M.R. Delbecq, et al. Photon-mediated interaction between distant quantum dot circuits, Nature Communications 4, Article number: 1400 (2013).

11 T. Frey, et al. Dipole Coupling of a Double Quantum Dot to a Microwave Resonator, Phys. Rev. Lett. 108, 046807 (2012).

12 K. D. Petersson, L. W. McFaul, M. D. Schroer, M. Jung, J. M. Taylor, A. A. Houck, and J. R. Petta, Circuit Quantum
Electrodynamics with a Spin Qubit, Nature 490, 380 (2012)

${ }^{13}$ H. Toida, T. Nakajima, and S. Komiyama, Vacuum Rabi Splitting in a Semiconductor Circuit QED System, Phys. Rev. Lett. 110, 066802 (2013).

14 J. Basset, et al. Single-electron double quantum dot dipolecoupled to a single photonic mode, Phys. Rev. B 88, 125312 (2013).

15 G.-W. Deng, et al. Circuit QED with a graphene double quantum dot and a reflection-line resonator, arXiv:1310.6118

16 G.-W. Deng et al., Coupling two distant double quantum dots to a microwave resonator, arXiv:1409.4980

17 J. J. Viennot, M. R. Delbecq, M. C. Dartiailh, A. Cottet, and T. Kontos, Out-of-equilibrium charge dynamics in a hybrid circuit quantum electrodynamics architecture, Phys. Rev. B 89, 165404 (2014).

18 M. D. Schroer, M. Jung, K. D. Petersson, and J. R. Petta, Radio Frequency Charge Parity Meter, Phys. Rev. Lett. 109, 166804 (2012).

19 Y.Y. Liu, K.D. Petersson,.J. Stehlik, J.M. Taylor, and J.R. Petta, Photon Emission from a Cavity-Coupled Double Quantum Dot, Phys. Rev. Lett. 113, 036801 (2014).

20 Y.-Y. Liu, J. Stehlik, C. Eichler, M. J. Gullans, J. M. Taylor, J. R. Petta, Semiconductor double quantum dot micromaser, Science 347, 285 (2015).

21 L. Childress, A. S. Sørensen, and M. D. Lukin, Mesoscopic cavity quantum electrodynamics with quantum dots, Phys. Rev. A 69, 042302 (2004).

22 A. Cottet, et al. Nanospintronics with carbon nanotubes, Semicond. Sci. Technol. 21, S78 (2006).

23 S. DeFranceschi, L. Kouwenhoven, C. Schönenberger, and W. Wernsdorfer, Hybrid superconductor-quantum dot devices, Nature Nanotechnology 5, 703 (2010).

24 M. Trif, V. N. Golovach, and D. Loss, Spin dynamics in InAs nanowire quantum dots coupled to a transmission line, Phys. Rev. B 77, 045434 (2008).

25 X. Hu, Y.-x Liu., and F. Nori, Strong coupling of a spin 
qubit to a superconducting stripline cavity, Phys. Rev. B 86, 035314 (2012).

26 C. Kloeffel, M. Trif, P. Stano, and D. Loss, Circuit QED with hole-spin qubits in Ge/Si nanowire quantum dots, Phys. Rev. B 88, 241405(R) (2013).

27 A. Cottet, and T. Kontos, Spin Quantum Bit with Ferromagnetic Contacts for Circuit QED, Phys. Rev. Lett. 105, 160502 (2010).

28 P.-Q. Jin, M. Marthaler, A. Shnirman, and G. Schön, Strong Coupling of Spin Qubits to a Transmission Line Resonator, Phys. Rev. Lett. 108, 190506 (2012).

29 X. Hu, Y.-x. Liu, F. Nori, Strong coupling of a spin qubit to a superconducting stripline cavity, Phys. Rev. B 86, 035314 (2012)

30 A. Cottet, C. Mora, and T. Kontos, Mesoscopic admittance of a double quantum dot, Phys. Rev. B 83, 121311(R) (2011)

31 P.-Q. Jin, M. Marthaler, J. H. Cole, A. Shnirman, and G. Schön, Lasing and transport in a quantum-dot resonator circuit, Phys. Rev. B 84, 035322 (2011).

32 M. J. Gullans, Y.-Y. Liu, J. Stehlik, J. R. Petta, J. M. Taylor, Phonon Assisted Gain in a Semiconductor Double Quantum Dot Maser, arXiv:1501.03499

33 C. Xu, and M. G. Vavilov, Quantum photovoltaic effect in double quantum dots, Phys. Rev. B 87, 035429 (2013).

${ }^{34}$ C. Xu, and M. G. Vavilov, Full counting statistics of photons emitted by a double quantum dot, Phys. Rev. B 88, 195307 (2013).

35 C. Bergenfeldt, and P. Samuelsson, Nonlocal transport properties of nanoscale conductor-microwave cavity systems, Phys. Rev. B 87, 195427 (2013).

${ }^{36}$ N. Lambert, C. Flindt, and F. Nori, Photon-mediated electron transport in hybrid circuit-QED, Europhys. Lett. 103, 17005 (2013).

37 L. D. Contreras-Pulido, C. Emary, T. Brandes, and R. Aguado, Non-equilibrium correlations and entanglement in a semiconductor hybrid circuit-QED system, New Journal of Physics 15, 095008 (2013).

38 C. Bergenfeldt, P. Samuelsson, B. Sothmann, C. Flindt, and M. Büttiker, Hybrid Microwave-Cavity Heat Engine, Phys. Rev. Lett. 112, 076803 (2014).

39 M. Kulkarni, O. Cotlet, and H. E. Türeci, Cavity-coupled double-quantum dot at finite bias: Analogy with lasers and beyond, Phys. Rev. B 90, 125402 (2014).

40 T. L. van den Berg, C. Bergenfeldt, and P. Samuelsson, Pump-probe scheme for electron-photon dynamics in hybrid conductor-cavity systems, Phys. Rev. B 90, 085416 (2014).

41 A. Cottet, T. Kontos, and A. Levy Yeyati, Subradiant Split Cooper Pairs, Phys. Rev. Lett. 108, 166803 (2012).

42 A. Cottet, Probing coherent Cooper pair splitting with cavity photons, Phys. Rev. B 90, 125139 (2014).

43 M. Schiró, and K. Le Hur, Tunable hybrid quantum electrodynamics from nonlinear electron transport, Phys. Rev. B 89, 195127 (2014).

${ }^{44}$ F. Hassler, A. R. Akhmerov and C. W. J. Beenakker, The top-transmon: a hybrid superconducting qubit for parityprotected quantum computation, New J. Phys. 13, 095004 (2011).

45 T. Hyart et al. Flux-controlled quantum computation with Majorana fermions, Phys. Rev. B 88, 035121 (2013).

46 C. Müller, J. Bourassa, and A. Blais, Detection and Manipulation of Majorana Fermions in Circuit QED, Phys. Rev. B 88, 235401 (2013)

47 Z.-Y. Xue et al., Tunable interfaces for realizing universal quantum computation with topological qubits, Phys. Rev. A 88, 024303 (2013).

48 E. Ginossar, and E. Grosfeld, Microwave transitions as a signature of coherent parity mixing effects in the Majoranatransmon qubit, Nat. Commun. 5, 4772 (2014).

49 C. Ohm and F. Hassler, Microwave readout of Majorana qubits, Phys. Rev. B 91, 085406 (2014).

50 K. Yavilberg, E. Ginossar, and E. Grosfeld, Fermion parity measurement and control in Majorana circuit quantum electrodynamics, arXiv:1411.5699

51 T. L. Schmidt, A. Nunnenkamp and C. Bruder, Majorana Qubit Rotations in Microwave Cavities, Phys. Rev. Lett. 110, 107006 (2013).

52 T. L. Schmidt, A. Nunnenkamp and C. Bruder, Microwave-controlled coupling of Majorana bound states, New J. Phys. 15, 025043 (2013).

53 J.-R. Souquet, M.J. Woolley, Julien Gabelli, Pascal Simon, Aashish A. Clerk, Photon-assisted tunneling with nonclassical light, Nature Communications 5, 5562 (2014).

54 C. Bergenfeldt and P. Samuelsson, Microwave quantum optics and electron transport through a metallic dot strongly coupled to a transmission line cavity, Phys. Rev. B 85, 045446 (2012).

${ }^{55}$ M. Trif, and Y. Tserkovnyak, Resonantly Tunable Majorana Polariton in a Microwave Cavity, Phys. Rev. Lett. 109, 257002 (2012).

56 A. Cottet, T. Kontos, and B. Douçot, Squeezing light with Majorana fermions, Phys. Rev. B 88, 195415 (2013).

57 B. Reulet, M. Ramin, H. Bouchiat, and D. Mailly, Dynamic Response of Isolated Aharonov-Bohm Rings Coupled to an Electromagnetic Resonator, Phys. Rev. Lett. 75, 124 (1995)

58 T. Holst, D. Esteve, C. Urbina, and M. H. Devoret, Effect of a Transmission Line Resonator on a Small Capacitance Tunnel Junction, Phys. Rev. Lett. 73, 3455 (1994).

59 A. Wallraff , A. Stockklauser, T. Ihn, J. R. Petta, and A. Blais, Comment on "Vacuum Rabi Splitting in a Semiconductor Circuit QED System", Phys. Rev. Lett. 111, 249701 (2013).

60 M. Büttiker, H. Thomas, and A. Prêtre, Mesoscopic capacitors, Phys. Lett. A 180, 364 (1993).

61 S. Nigg, R. Lopez, and M. Büttiker, Mesoscopic Charge Relaxation, Phys. Rev. Lett. 97, 206804 (2006).

62 S. Nigg, and M. Büttiker, Quantum to Classical Transition of the Charge Relaxation Resistance of a Mesoscopic Capacitor, Phys. Rev. B 77, 085312 (2008).

63 C. Mora, and K. Le Hur, Universal Resistances of the Quantum RC circuit, Nat. Physics 6, 697 (2010).

64 Y. Hamamoto, T. Jonckheere, T. Kato, and T. Martin, Dynamic response of a mesoscopic capacitor in the presence of strong electron interactions, Phys. Rev. B 81, 153305 (2010).

65 M. Filippone, K. Le Hur, and C. Mora, Giant Charge Relaxation Resistance in the Anderson Model, Phys. Rev. Lett. 107, 176601 (2011).

66 A. Golub, and E. Grosfeld, Charge resistance in a Majorana $R C$ circuit, Phys. Rev. B 86, 241105(R) (2012).

67 J. Klinovaja, M. J. Schmidt, B. Braunecker, and D. Loss, Carbon nanotubes in electric and magnetic fields, Phys. Rev. B 84, 085452 (2011).

68 See e.g. Single Charge Tunneling: Coulomb Blockade Phenomena in Nanostructures, H. Grabert and M. H. Devoret (eds.), NATO ASI Series B 234 (Plenum, New York, 1992).

69 W. V. D. Hodge, The Theory and Applications of Har- 
monic Integrals, Cambridge University Press (1941).

70 P. Griffiths and J. Harris, Principles of Algebraic Geometry, Wiley Interscience, coll.Wiley Classics Library (1994).

71 Y. Kubo, et al. Strong Coupling of a Spin Ensemble to a Superconducting Resonator, Phys. Rev. Lett. 105, 140502 (2010).

72 D. I. Schuster, et al. High-Cooperativity Coupling of Electron-Spin Ensembles to Superconducting Cavities, Phys. Rev. Lett. 105, 140501 (2010).

73 Z.-L. Xiang, S. Ashhab, J. Q. You, F. Nori, Reviews of Modern Physics 85, 623 (2013).

${ }^{74}$ P. Nataf and C. Ciuti, No-go theorem for superradiant quantum phase transitions in cavity QED and counterexample in circuit QED, Nature Commun. 1, 72 (2010).

75 O. Viehmann, J. von Delft, and F. Marquardt, Superradiant Phase Transitions and the Standard Description of Circuit QED, Phys. Rev. Lett. 107, 113602 (2011)

76 L. P. Kouwenhoven, et al. Observation of Photon-Assisted Tunneling through a Quantum Dot, Phys. Rev. Lett. 73, 3443 (1994).

77 G. Platero, and R. Aguado, Photon-assisted transport in semiconductor nanostructures, Physics Reports 395, 1 (2004).

78 J. R. Schrieffer, Theory of Electron Tunneling, Rev. Mod. Phys. 36, 200 (1964).

79 J. Fransson, O. Eriksson, and I. Sandalov, Effects of non-orthogonality and electron correlations on the timedependent current through quantum dots, Phys. Rev. B 66, 195319 (2002).

80 R. E. Prange, Tunneling from a Many-Particle Point of View, Phys. Rev. 131, 1083 (1963).

81 Strictly speaking, this assumption is not valid for non ergodic reservoirs such as integrable billiards or Anderson localized systems. Our theory can be applied to these cases provided one keeps the $j$ dependence of the coupling parameter $\alpha_{o j}$.

82 A. Vukics, T. Grießer, and P. Domokos, Elimination of the A-Square Problem from Cavity QED, Phys. Rev. Lett. 112, 073601 (2014).

83 V. Mourik et al., Signatures of Majorana Fermions in Hybrid Superconductor-Semiconductor Nanowire Devices, Science 3361003 (2012)

${ }^{84}$ L. Bretheau, Ç. Ö. Girit, Hugues Pothier, D. Esteve, and C. Urbina, Exciting Andreev pairs in a superconducting atomic contact, Nature 499, 7458 (2013) 312

85 The results of section IIE can be generalized straighforwardly to d-wave or p-wave superconductivity by using a non-local gap term in $\Delta\left(\vec{r}, \vec{r}^{\prime}\right) e^{\hat{\Phi}(\vec{r})+\hat{\Phi}\left(\vec{r}^{\prime}\right)}$ in Eq.(16), with a different structure in spin-space in case of p-wave superconductivity.

86 P. G. de Gennes, Superconductivity of Metals and Alloys (Benjamin, New York, 1966).

87 M. Leijnse, and K. Flensberg, Introduction to topological superconductivity and Majorana fermions, Semicond. Sci. Technol. 27, 124003 (2012).

88 J. Alicea, New directions in the pursuit of Majorana fermions in solid state systems, Rep. Prog. Phys. 75, 076501 (2012).

89 C.W.J. Beenakker, Search for Majorana Fermions in Superconductors, Annu. Rev. Con. Mat. Phys. 4, 113 (2013).

90 J. J. Viennot, J. Palomo, and T. Kontos, Stamping single wall nanotubes for circuit quantum electrodynamics, Appl. Phys. Lett. 104, 113108 (2014).

91 J. Stehlik et al., Fast charge sensing of a cavity-coupled double quantum dot using a Josephson parametric amplifier, arXiv:1502.01283

92 W. Liang et al., Fabry - Perot interference in a nanotube electron waveguide, Nature 411, 665 (2001).

93 J. Gabelli, G. Fève, J.-M. Berroir, B. Plaçais, A. Cavanna, B. Etienne, Y. Jin, and D. C. Glattli, Violation of Kirchhoff's Laws for a Coherent RC Circuit, Science 313, 499 (2006).

94 H. Shiba, The Korringa Relation for the Impurity Nuclear Spin-Lattice Relaxation in Dilute Kondo Alloys, Prog. Theor. Phys. 54, 967 (1975).

95 J. Gabelli, Mise en évidence de la cohérence quantique des conducteurs en régime dynamique, <tel-00011619>, Thesis of the Université Pierre et Marie Curie - Paris VI (2006).

96 A. Sarlette, J. M. Raimond, M. Brune, and P. Rouchon, Phys. Rev. Lett. 107, 010402 (2011).

97 E. T. Holland et al., Single-photon Resolved Cross-Kerr Interaction for Autonomous Stabilization of Photon-number States, arXiv:1504.03382

98 J. D. Jackson, Classical Electrodynamics. New York: John Wiley and Sons (1962).

99 S. E. Nigg et al. Black-Box Superconducting Circuit Quantization, Phys. Rev. Lett. 108, 240502 (2012). 